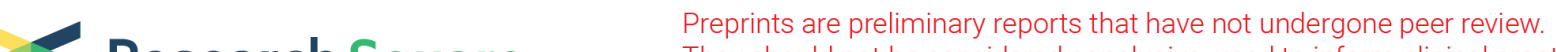 Research Square They should not be considered conclusive, used to inform clinical practice, or referenced by the media as validated information.
}

\section{Piezo1 Promoted Hepatocellular Carcinoma Progression and EMT Through Activating TGF- $\beta$ Signaling by Recruiting Rab5c}

\section{Yiming Li}

Xiangya Hospital Central South University

\section{Cong $\mathrm{Xu}$}

Xiangya Hospital Central South University

\section{Bo Sun}

Xiangya Hospital Central South University

Fangjing Zhong

Xiangya Hospital Central South University

\section{Momo Cao}

Xiangya Hospital Central South University

Lianyue Yang ( $\square$ lianyueyang@csu.edu.cn )

Xiangya Hospital Central South University https://orcid.org/0000-0002-8805-872X

\section{Primary research}

Keywords: Piezo1, Hepatocellular Carcinoma, Prognosis, Cancer Progression, TGF- $\beta$ signaling, EMT

Posted Date: November 23rd, 2021

DOI: https://doi.org/10.21203/rs.3.rs-1057169/v1

License: (1) This work is licensed under a Creative Commons Attribution 4.0 International License. Read Full License

Version of Record: A version of this preprint was published at Cancer Cell International on April 23rd, 2022. See the published version at https://doi.org/10.1186/s12935-022-02574-2. 


\section{Abstract \\ Background}

Piezo1 plays critical roles in vascular development during early embryogenesis. However, the function of Piezo1 in hepatocellular carcinoma (HCC) remain elusive. This study aimed to explore the function and mechanisms of Piezo1 in HCC.

\section{Methods}

qRT-PCR, western blot and immunohistochemistry analyses were used to determine Piezo1 expression in HCC samples and cell lines. The clinical significance of Piezo1 was assessed in two independent study cohorts containing 280 patients with HCC. Gene set enrichment analysis (GSEA) was performed to explore the signaling pathway of Piezo1. A series of in vitro and in vivo experiments were used to determine the role and molecular mechanism of Piezo1 in HCC progression.

\section{Results}

Piezo1 expression was significantly upregulated in HCC tissues and cell lines. High Piezo1 expression was closely correlated with aggressive clinicopathological features, poor clinical outcomes of HCC patients. Moreover, knockdown of Piezo1 in HCCLM3 and Hep3B cells significantly inhibited proliferation, migration, invasion and epithelial-mesenchymal transition (EMT) of HCC cells in vitro, and tumor growth, EMT and metastasis in vivo. Further mechanism study indicated that these phenotypic and function changes were mediated by TGF- $\beta$ signaling pathway. Finally, we proved that Piezo1 exerted its tumor promotion effect by recruiting Rab5c to activating TGF- $\beta$ signaling pathway.

\section{Conclusions}

We proved Piezo1 promotes progression of hepatocellular carcinoma via TGF- $\beta$ signaling, which may serve as a novel prognostic predictor and the potential therapeutic target for HCC patients.

\section{Introduction}

Hepatocellular carcinoma (HCC) ranks as the sixth most common malignant tumor and the fourth leading cause of cancer death worldwide, and China is the most high risk HCC area[1]. Even multiple therapeutic measures have been proposed in newly presented guidelines of HCC in China [2], but liver resection remains the most effective approach for HCC. Although its mortality decreased along with advances in surgical resection, the long-term outcome and effect of medicine remain unsatisfactory [3]. The 5-year survival rate in HCC patients beyond the Milan criteria after surgical resection is only $30-50 \%$ $[4,5]$, mainly due to the high frequency of recurrence and metastasis. Comprehensive understanding of 
the molecular mechanisms underlying the Progression of HCC is crucial for its prevention, diagnosis and treatment [6]. Up to now, many factors have been identified to play important role in the progress of HCC, including aberrantly expressed miRNAs, LncRNAs and proteins, and has been suggested to be the HCC prognostic or diagnostic markers [7-10]. Although remarkable improvements had been done by global scientists. However, the molecular mechanisms of HCC progression remain largely unclear.

The Piezo family are known as mechanosensitive cation selective channels includes two isoforms, Piezo1 and Piezo2[11]. Piezo1 is widely expressed in numerous mammalian tissues with particularly high in lung, bladder and skin [12-14]. In addition to be a mechano-sensors and converts environmental signals into intracellular $\mathrm{Ca}^{2+}$ responses, and involved in vascular development and function [15]. Recent studies have addressed that Piezo1 also have multiple functions, in regulating the cardiac macrovascular development during early embryogenesis, control rapid epithelial cell division, lymphatic valve formation and altered neuron stem cells differentiation [16-19]. So far, Piezo1 were involved in proliferation and migration in gastric cancer, breast cancer, synovial sarcoma and glioma [20-23], but the function and mechanism of Piezo1 in HCC remain poorly elucidated. In our preliminary study, Piezo1 highly expressed in HCC tissue and cell lines, but not Piezo2. Thus, those studies led to a hypothesis that Piezo1 plays an important role in HCC progression.

In this study, the function of Piezo1 was explored in HCC and found that high expression of Piezo1 is closely correlated with poor prognosis of HCC patients. We also confirmed that Piezo1 promotes HCC progression through EMT. Mechanism studies show that Piezo1 could recruits and activates Rab5c in HCC, which promoted the phosphorylation of Smad2/3 and triggers classical TGF- $\beta$ signaling pathway in HCC. Thus, Piezo1 might serve as a potential prognostic biomarker and therapeutic target for HCC.

\section{Materials And Methods}

\section{HCC samples and patients}

A total of $150 \mathrm{HCC}$ specimens in training cohort collected from January 2009 to December 2012 were randomly selected from the patients received liver resection at Department of Surgery, Xiangya Hospital of Central South University. Another $130 \mathrm{HCC}$ specimens in validation cohort collected from January 2010 to December 2012 were randomly selected from the Department of Abdominal Surgical Oncology, Affiliated Cancer Hospital of Xiangya School of Medicine, Central South University. The patient demographics and clinicopathological variables of the two cohorts are described in Supplementary Table S1. Furthermore, 30 matched fresh HCC tissues and adjacent nontumoral liver tissues (ANLTs) were collected from Xiangya Hospital from September to December 2019. 10 fresh HCC tissues in each clinic subtypes were collected from Xiangya Hospital from June to December 2019. The diagnosis of HCC in all patients was confirmed by two independent histopathologists. The present study was approved by the Ethics Committee of Xiangya Hospital, Central South University. All patients and their families provided written informed consent and agreed to the use of their tissue samples in the study in accordance with the Declaration of Helsinki. 


\section{Extraction of Membrane and cytosol fraction}

Cells were washed 3 times by PBS and added lysis buffer ( $250 \mathrm{mM}$ sucrose, $10 \mathrm{mM}$ HEPES, 1 mM EDTA, $\mathrm{pH}$ 7.5) supplemented with protease inhibitors, and scraped into centrifuge tube. Extracts were mechanically lysed 50times by homogeniser, and subjected to $90 \mathrm{~min}$ of ultracentrifugation at 45,000 rpm (Thermo Fisher Scientific, Waltham, MA) at $4^{\circ} \mathrm{C}$. The resulting supernatant was the cytosolic fraction. The membrane fraction was resuspended in $100 \mu \mathrm{l}$ immunoprecipitation assay buffer $(10 \mathrm{mM}$ Tris- $\mathrm{HCl}$, pH 7.5, 1 mM EDTA, 0.5 mM EGTA, 1\% Triton X-100, 0.1\% sodium deoxycholate, $0.1 \%$ SDS $140 \mathrm{mM} \mathrm{NaCl}$ ), with protease inhibitors. Cytosol and membrane protein bands were quantified and relativized against their respective fraction markers RhoGDI and transferrin receptor [24].

\section{Immunohistochemical (IHC) analysis and scoring}

Immunohistochemical staining on formalin-fixed, paraffin-embedded tissue sections $4 \mu$ minthickness wasperformed using the polymer HRP detection system (Zhongshan Goldenbridge Biotechnology, Beijing, China). Immunohistochemical experiments were conducted as previously described $[9,25]$. The IHC score of target proteins was independently evaluated by two investigators according to the proportion and intensity of positive cells within five randomly selected fields per slide (magnification, $\times 400$ ). The intensity was assessed by four grades: 0 for none; 1 for weak; 2 for moderate; 3 for strong. The percentage of positive cells was divided into five degrees: 0 , no positive tumor cells; 1 for $\leq 5 \%$; 2 for 6 $25 \%$; 3 for $26-75 \% ; 4$ for $\geq 76 \%$. Immunoreactive score was calculated by multiplying the staining extent score with the intensity score. As previous reported, high expression was defined as a staining index score $>4$, while low expression was defined as a staining index score $\leq 4[26,27]$.

\section{HCC mouse models}

Animal xenograft assays were conducted with 6-weekold male BALB/c nude mice (six mice per group). $5 \times 10^{6}$ indicated cells were subcutaneously injected into the right dorsal flank of nude mice. Tumor sizes were measured at the indicated time points and calculated with the following formula: Tumor volume $=\mathrm{L}$ $\times W^{2} \times 0.5$ (L, length; W, width) [28]. After 4 weeks, the mice were sacrificed, and the tumors were harvested to weigh and undergo further experiments. Orthotopic tumor implantation was performed as described previously [29]. After 8 weeks, the mice were sacrificed, and the livers and lungs were harvested, the tumor size was measured by the vernier caliper as previously described [7], imaged and processed for histopathological examination. All animal experiments were conducted at the Animal Institute of CSU according to the protocols approved by the Medical Experimental Animal Care Commission of CSU.

\section{Statistical analysis}

Statistical analysis was performed using SPSS 24.0 software (SPSS Inc., Chicago, IL). The experimental data was presented as the mean \pm SD and analyzed using Student's $t$ test or one-way ANOVA. The Chi- 
squared test was applied to examine the association between Piezo1 expression and clinicopathological parameters. Survival curves for patients were calculated using the Kaplan-Meier method and analyzed using the log-rank test. Prognostic factors were examined by univariate and multivariate analyses using the Cox proportional hazards model. Spearman's rank analysis was performed to determine the correlation between different protein levels. One-sample $t$-test or Wilcoxon signed-rank test was performed to measure the membrane and cytolic protein expression level. All differences were deemed statistically significant at $P<0.05$.

Further details of materials and methods are described in the Supplementary Materials and Methods.

\section{Result}

\section{Piezo1 is significantly upregulated in HCC cell lines and tissues}

Firstly, we examined the levels of Piezo1 and Piezo2 mRNA in 7 HCC cell lines and 2 normal hepatocytes, the Primary human hepatocytes (PHH) and immortalized hepatocytes (L02) (Fig. 1 A). Notably, the expression of Piezo1 mRNA in HCC cells significantly stronger than PHH and L02. But this difference of expression was not observed in Piezo2(Fig. 1 B). Then, we also detected the mRNA expression of Piezo1 and Piezo2 in frozen HCC tissue and the corresponding adjusted nontumor liver tissue (ANLT). Consistent with the cell lines, the Piezo1 mRNA expression level in HCC tissue were significantly higher than ANLT. Previously, we found a specific subtype of HCC in which the tumor was greater than $5 \mathrm{~cm}$ in diameter with a single lesion, but the tumor grew expansively with an intact capsule or pseudocapsule. We named this special subtype as SLHCC (solitary large hepatocellular carcinoma), and divided HCC into three different subtypes: SLHCC, nodular HCC (NHCC, node number >1), and small HCC (SHCC, tumor diameter $\leq 5 \mathrm{~cm}$ ) [4]. We demonstrated that SLHCC possessed unique clinicopathological and molecular pathological characteristics and exhibited a similar long-term overall and disease-free survival with SHCC, but much better than NHCC [4,7-9]. We also detected mRNA of Piezo1 in the 3 clinic subtypes of HCC, and the NHCC with high metastatic potentials expressed relatively higher level of Piezo1 than the SLHCC and SHCC with low metastatic potentials, but Piezo2 was not significantly differential expressed. Then, Piezo1 and Piezo2 protein expression in HCC cell lines and PHH, L02 cells was detected by WB (Fig. 1 CD). Therefore, we supposed that Piezo1 might associate with progression of HCC, but not Piezo2. Expression level of Piezo1 was also analyzed by immunohistochemistry (IHC), which suggested that Piezo1 protein was highly expressed in HCC (Fig. 1 E). These data reveal that Piezo1 upregulated in HCC cell lines and tissues, and indicates that it's very necessary to explore the role of Piezo1 in HCC progression.

\section{Upregulated Piezo1 is associated with HCC poor prognosis}


To further determine the expression level of Piezo1 in HCC, the analyses of mRNA datasets from TCGA and Gene Expression Omnibus (Fig. 2 A, TCGA, GSE76297, GSE36376, GSE10143) revealed that Piezo1 upregulated in HCC. Then, to explore the clinical significance of Piezo1 expression level in HCC, we analyzed Piezo1 expression level by IHC (Fig. 2 B) and studied the association of Piezo1 expression with clinicopathological features and survival of HCC patients using the training cohort $(n=150)$ and validation cohort $(n=130)$ (Supplementary Table S1). In the training cohort, a high expression level of Piezo1 was closely correlated with tumor size, tumor nodular number, capsulation formation, micro- and macrovascular invasion, high Edmondson-Steiner grade, advanced tumor node metastasis stage (TNM), Barcelona Clinic Liver Cancer (BCLC) stage and China Clinic Liver Cancer (CNLC) stage[2] (all $P<0.05$, Table 1 ). Furthermore, multivariate analysis revealed that high Piezo1 expression was an independent risk factor for both OS and DFS of HCC patients after liver resection (Table 2). Piezo1 was further verified as an independent prognosis marker, and its high expression was associated with poor survival of HCC patients $囚$ this result was further verified in validation cohort (Supplementary Table S2). In addition, Kaplan-Meier survival analysis in TCGA showed that HCC patients with low Piezo1 had longer OS and DFS, but Piezo2 showed no significance between low- and high- expression group (Fig. 2 C), which consistent with our above research. Notably, survival analysis for training cohort reveals that HCC patients in the high Piezo1 expression group had shorter OS (1-, 3-, and 5-year OS: 91.33\%, 67.33\%, and 48.67\% vs. $79.33 \%, 41.33 \%$, and $21.33 \%$ ) and DFS rates (1-, 3-, and 5-year DFS: $82.31 \%, 61.54 \%$, and $41.54 \%$ vs. $70.77 \%, 31.54 \%$, and $10.77 \%$ ) than patients in the low-expression group (Fig. 2 D). Then, survival analysis for the integrated cohort combined by training and validation cohort demonstrated high Piezo1 expression level indicates shorter OS and DFS in HCC patients (Fig. 2 E). Besides, this result was also observed in 3 clinical subtypes of HCC (Fig. 2 E). These data fully confirmed that Piezo1 was closely correlated with poor survival and could be used as a novel independent prognosis biomarker for HCC patients after hepatic resection.

\section{Piezo1 promotes HCC proliferation囚invasion and metastasis in vitro and in vivo}

To understand the function of Piezo1 in HCC cells, we manipulated Piezo1 expression in cells by short hairpin RNA (shRNA). Due to the molecular weight of Piezo1 is too large to construct overexpression vector, we interfered Piezo1 in two highest expression cell lines HCCLM3 and Hep3B cells by three shRNAs separately, as named HCCLM3 ${ }^{\text {shPiezo1 }}$ and Hep3B ${ }^{\text {shPiezo1 }}$. The corresponding control lentivirus

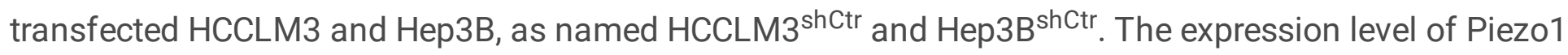
was identified by real-time PCR and western blotting. shRNA2 was the most effective one and was chosen for further study (Supplementary Fig. S1A, B). The wound-healing and transwell assays were used to investigate migration and invasion capacity. The results showed that $\mathrm{HCCLM} 3^{\text {shCtr }}$ cells had a faster wound closure rate and more invasion than HCCLM3 ${ }^{\text {shPiezo1 }}$ cells, and Hep3B ${ }^{\text {shPiezo1 }}$ cells had also markedly reduced migratory and invasive capacity (Fig. 3 A, B). Compared to HCCLM3 ${ }^{\text {shPiezo1 }}$, cell clone formation assay (Fig. 3C) and MTT assay (Supplementary Fig. S2) indicated HCCLM3 ${ }^{\text {shCtr }}$ had a higher 
proliferation rate. Consistently, Hep3B ${ }^{\text {shCtr }}$ cells also formed more colonies in colony formation assay. In IF stained F-actin (Fig. 3 D), we also noticed the morphological change when Piezo1 was interfered, in which the spindle-like mesenchymal morphology of HCCLM3 ${ }^{\text {shCtr }}$ and Hep3B ${ }^{\text {shCtr }}$ changed into cobblestone-like epithelial morphology of HCCLM3 ${ }^{\text {shPiezo1 }}$ cells and Hep3B ${ }^{\text {shPiezo1 }}$ cells. To verify the above findings in vivo, we established subcutaneous (SC) xenograft tumor and orthotopic xenograft tumor models, as previously described[30]. After 6 weeks, $\mathrm{HCCLM}^{\text {shCtr }}$ and Hep3B ${ }^{\text {shCtr }}$ cell-derived tumors at the SC implantation sites were larger and grew more rapidly than HCCLM3 ${ }^{\text {shPiezo1 }}$ and Hep3B ${ }^{\text {shPiezo1 }}$ derived tumors (Fig. $3 \mathrm{E}$ ). Consistently, liver orthotopic xenograft tumor also showed that $\mathrm{HCCLM}^{\text {shPiezo1 }}$ and Hep3B ${ }^{\text {shPiezo1 }}$ derived tumors significantly smaller than $\mathrm{HCCLM}^{\mathrm{NC}}$ and $\mathrm{Hep} \mathrm{B}^{\mathrm{NC}}{ }_{-}$ derived tumors, indicates that Piezo1 knockdown inhibited tumor growth in vivo (Fig. $3 \mathrm{~F}$ ). Taking these results together, our results shows that Piezo1 promotes HCC growth, progression in vitro and in vivo.

\section{Piezo1 promotes EMT in HCC}

In the present study, the morphological changes in IF stained F-actin attracted our attention when we silenced Piezo1 expression in HCCLM3 and Hep3B cells. We speculated that Piezo1 could induce EMT for HCC cell lines. To confirm our hypothesis, we first analyzed the relationship between Piezo1 expression and hallmarks of EMT in HCC patients from the TCGA database with gene set enrichment analysis (GSEA). Piezo1 highly expression was significantly correlated with the hallmarks of EMT (NES $=1.678$, NOM $p$-val=0.004, Fig. 4 A). Then, the expression relationship between Piezo1 and EMT markers was analyzed in cytological and histological experiments. At the protein level, Piezo1 knockdown in HCCLM3 and Hep3B cells resulted in increased the expression of E-cadherin and decreased the expression of vimentin, whereas high Piezo1 expressed HCCLM3 ${ }^{\text {shCtr }}$ and Hep3B ${ }^{\text {shCtr }}$ cells resulted in opposing results (Fig. 4 B). IF analysis showed that the expression of the mesenchymal marker vimentin was significantly reduced in Piezo1 interfered HCCLM3 ${ }^{\text {shPiezo1 }}$ and Hep3B ${ }^{\text {shPiezo1 }}$ cells and increased the expression of the epithelial marker E-cadherin, while relatively high expression level of Piezo1 induced inverse results in $\mathrm{HCCLM}^{\text {shCtr }}$ and Hep3B ${ }^{\text {shCtr }}$ (Fig. $4 \mathrm{C}$ ). IHC staining of consecutive HCC sections also showed that vimentin expression levels were up-regulated in high Piezo1-expressing cell-derived tumors, whereas Ecadherin expression was reduced (Fig. 4 D). Moreover, IHC revealed that Piezo1 expression was negatively correlated with E-cadherin expression and positively correlated with vimentin expression (Figure 4E). These results indicated that Piezo1 promotes EMT in HCC cell lines.

\section{Piezo1 promote HCC progression via TGF- $\beta$ signaling}

In order to investigate the mechanism of Piezo1 induce EMT and promotes progression of HCC, we systemically screened the potential signaling pathways manipulated by Piezo1 in HCC. First, we analyzed the information of HCC patients from the TCGA database using Gene set enrichment analysis (GSEA). TGF- $\beta$ signaling was found to be the most significantly enriched pathway (NES $=1.775$, NOM $p$ - 
val=0.002, Fig. 5 A. The result of GSEA shown in Supplementary Table S3,S4). Then, a Cignal Finder Cancer 10-Pathway Reporter Array was performed in HCC cells with manipulated Piezo1 expression to further screen and confirm the signaling pathway regulated by Piezo1. The results showed that TGF$\beta$ signaling was the most significantly altered pathway (Fig. $5 \mathrm{~A}$ ). So, we further investigated the effect of Piezo1 on the expression of key members of the TGF- $\beta$ signaling pathway and observed that in shPiezo1 cells, the protein levels of AKT, p-AKT, ERK, $p$-ERK and smad2/3 had no significant difference, only phosphorylated-smad2/3 was down-regulated in Piezo1 knockdown cells (Fig. 5 B). Meanwhile, several genes related to EMT were analyzed. The data showed that E-cadherin was up-regulated and vimentin was down-regulated in Piezo1 knockdown cells. Moreover, IHC staining in $40 \mathrm{HCC}$ samples randomly selected from the training and validation cohorts showed that $\mathrm{p}-\mathrm{Smad} 2 / 3$ protein was highly expressed in HCC tissues and positively correlated with Piezo1 expression $(P<0.001, r=0.578$, Fig. $5 \mathrm{C})$. Then, the four

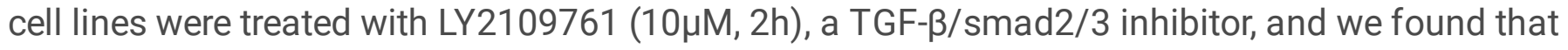
LY2109761 did not affected Piezo1 expression. Compared with the control cells, HCCLM3 ${ }^{\text {shPiezo1 }}$ and Hep3B ${ }^{\text {shPiezo1 }}$ cells showed a decreasing trend in phosphorylation level of Smad2/3, which was similar to LY2109761 treated HCCLM $3^{\text {shCtr }}$ and Hep3B ${ }^{\text {shCtr }}$ cells. It proves that Piezo1 activates TGF- $\beta$ signaling in HCC cells (Fig. 5 D). To further study the role of Piezo1 and TGF- $\beta$ signaling in progression, Woundhealing (Fig.5 E) and transwell (Fig. 5 F) assays showed that LY2109761 treatment obviously decreased the migration and invasion capacity of Piezo1 high expressed cells, but had none or less effect for cells in HCCLM3 ${ }^{\text {shPiezo1 }}$ and Hep3B ${ }^{\text {shPiezo1 }}$ cells which Piezo1 expression and TGF- $\beta$ signaling activity was low. Taken together, the public database analysis and our results showed that Piezo1 could activate the canonical TGF- $\beta$ / Smad2/3 signaling induced EMT in HCC and, promoting HCC progression.

\section{Piezo1 activates TGF- $\beta /$ Smad2/3 signaling by recruiting Rab5c}

The above results revealed that Piezo1 promote HCC progression via activating the TGF- $\beta$ signaling, to further explore the molecular mechanism by which Piezo1 exerted the functions in HCC. In consideration of that Piezo1 is a stretch-activated ion channel and the activation of Piezo1 channels increased the intracellular $\mathrm{Ca}^{2+}$ concentration, we treated HCCLM3 and Hep3B cells with Yoda1 (the activator of Piezo1, $20 \mathrm{mM}$ ) to increase the intracellular $\mathrm{Ca}^{2+}$ concentration and GsMTx4 (the inhibitor of Piezo1, $2.5 \mu \mathrm{M}$ ) to decrease $\mathrm{Ca}^{2+}$ concentration [14] [31], then examined p-Smad2/3 and marker of EMT, found that $\mathrm{Ca}^{2+}$ influx was not the dominant factor in Piezo1 activated TGF- $\beta$ signaling(Supplementary Fig. S3 A, B). Then we reviewed literatures and searched BioGrid 4.4 database (Supplementary Fig. S3 C), found that Piezo1 might interact with Rab5c, an isoform of Rab5, which belongs to the small GTPases of Rab family. Double IF revealed the colocalization of Piezo1 and Rab5c in HCCLM3 ${ }^{\mathrm{NC}}$ and Hep3B ${ }^{\mathrm{NC}}$ cells. More than that, we also observed that the Rab5c has a tendency to enriched in membrane when Piezo1 highly expressed (Fig. 6 A). Then, co-IP results revealed Piezo1 and Rab5c could interact with each other in HCC cells (Fig. 6 B, C). Next, we investigated whether the interaction between Piezo1 and Rab5c could affect rab5c expression in HCC cells. Interestingly, we found that silenced Piezo1 in HCCLM3 and Hep3B cells 
did not changed the protein expression level of Rab5c, but the phosphorylation level of Smad2/3 and expression level of EMT markers have changed correspondingly (Fig. 6 D). Combined with the location of Rab5c in the IF result, we further analyzed membrane enrichment of Rab5c by western blotting, and confirmed that Rab5c was significantly enriched in membrane fractions in cells highly expressed Piezo1(Fig. 6 E). Summarily, these results indicate that Piezo1 activates TGF- $\beta /$ Smad2/3 signaling by recruiting Rab5c without change the total expression level of Rab5c.

\section{Piezo1 mediated activating of TGF- $\beta$ signaling and promote HCC progression and EMT through Rab5c}

It has proved that Piezo1 activates TGF- $\beta$ signaling by recruiting Rab5c in the level of protein, but the function of Rab5c in this progress still unknown. Next, we need to test whether Rab5c is indispensable for Piezo1-mediated activation of TGF- $\beta$ signaling and promotion of HCC progression and EMT. we transfected Rab5c in HCCLM3 ${ }^{\text {shPiezo1 }}$ and Hep3B ${ }^{\text {shPiezo1 }}$ cells. MTT, wound healing and transwell assay results revealed overexpression of Rab5c reversed the down-regulation of the invasion, migration and proliferation potential caused by Piezo1 silencing in HCCLM3 ${ }^{\text {shPiezo1 }}$ and Hep3B ${ }^{\text {shPiezo1 }}$ cells (Fig. 7 A-C). The morphology and expression of EMT marker also reversed along with the overexpression of Rab5c in Piezo1 interfered HCC cells (Fig. 7 D, E). In summary, the results indicate that Piezo1 promote HCC progression by activating TGF- $\beta$ signaling via recruiting Rab5c (Fig. 7 F).

\section{Discussion}

Recently, considerable achievements have made in the diagnosis and treatment of HCC, such as the advances in surgical treatment, precise treatment and immunological therapy, prolonged survival time of HCC patients to some extent, but invasion and metastasis are still main reason for cancer associated death in HCC. The clinical outcome in HCC patients remains far from satisfactory because of the invasion and metastasis. Thus, it is still critical to gain a better understanding of the mechanisms underlying HCC progression.

Piezo proteins are considered to be large integral membrane proteins with 24-40 transmembrane domains, making them the proteins with the largest number of transmembrane domains[11,32, 33], and proved as central in diverse biological processes, including cardiovascular development and cancer progression $[16,22,34]$. Piezo1 has been previously verified to be a regulator of various key biological processes, including cell division, migration, and differentiation [35]. But in cancer, the role of Piezo1 is controversial. It has been proved that Piezo1 was downregulated in small lung cancer cell lines, therefore Piezo1 might be a cancer suppressor which suggested to inhibit the cells migration and distant metastases in lung cancer in lung cancer [36]. On the contrary, more reports reminded that Piezo1 might function as an oncogene-related molecule in several types of cancer [21, 23, 37-39]. Piezo1 is highly expressed in the cytoplasm of human prostate carcinoma tissue [40]. In oral squamous cell carcinoma, elevated Piezo1 induced by YAP signaling was required for cell proliferation [38]. Piezo1 is also a vital 
regulator of innate immune responses and targeting Piezo1 in myeloid cells is protective against cancer with a reduced infiltrate of immune cells [35].

Consistent with majority researches and TCGA, GEO database, we confirmed Piezo1 high expression indicated the poor prognosis in HCC patients after liver resection. Notably, the expression level of Piezo1 was proved to also an independent risk factor for overall survival and disease-free survival of HCC patients. Then, we proved that Piezo1 was associated with poor prognosis of HCC patients. These findings implicate that Piezo1 has potential to serve as an independent prognostic marker for HCC patients after liver resection, which might facilitate precision medicine, helping to predict the prognosis, direct the individualized therapy or act as a therapeutic target.

In order to explore the expression differences for different clinical subtypes of HCC, we have tested the Piezo1 expression level in the 3 subtypes of HCC. It's worth noting that Piezo1 are differently expressed in SLHCC, SHCC and NHCC, the results were also consistent with our previous studies that different clinical HCC subtypes had distinct molecular characteristics [4, 30, 41-45], and Piezo1 might be an marker of molecular subtyping after a large size validation. The functional experiments also revealed that Piezo1 knockdown could inhibit progression of HCC, which identified the function of Piezo1 in promoting HCC aggression. Although Piezo1 proved that might activate various pathways, such as Akt/mTOR pathway [37],MT1-MMP/MMP2 signaling pathway[46], HIF-1a-VEGF signaling pathway[39]. Our research showed that Piezo1 activated TGF- $\beta$ signaling which has not been reported, indicated Piezo1 might be a regulator of multi signaling pathways, and functioning through different pathways in various types of cancers.

In our research in virto, we have noticed the morphologic change of the shPiezo1 cells, and the GSEA report also reminded us the correlation of Piezo1 and EMT, then we guess that Piezo1 might play a role in EMT of HCC, and the follow-up experiment results established our hypothesis. EMT is one of the key mechanisms of TGF- $\beta$ signaling regulating cancer progression [47-49]. Lately, one research has proved that downregulated Piezo1 could impairs HCC growth via deregulation of the MAPK-mediated YAP signaling pathway in HepG2 cell line and in Vivo [50]. Unlikely, our research revealed the prognostic value of Piezo1 and more concern about invasion and migration of HCC, and GSEA in our research indicates that EMT and TGF- $\beta$ signaling regulated by Piezo1, but not MAPK or YAP signaling, and we also verified it in clinical specimens. The difference indicates the complex function of Piezo1.

As reported, tumor cells that have lost the cytostatic response may undergo epithelial-to mesenchymal transition (EMT) in response to TGF- $\beta$ signaling and become more invasive[51]. As one of the hallmarks of signaling pathways that regulate cancer progression, TGF- $\beta$ signaling induces tumor proliferation and metastasis [52,53], which also validated in HCC cells of our research. Members of the TGF- $\beta$ family control numerous cellular functions including proliferation, migration, apoptosis, differentiation, and EMT[54]. Consistent with reports, we found that TGF- $\beta$ signaling and EMT were both enriched in Piezo1 high group in GSEA, and the result of 10-Pathway Reporter Array further confirmed the GSEA results. Subsequently, we found that Piezo1 knockdown had the equal blockage effect as the specific inhibitor of 
TGF- $\beta$ signaling, LY2109761. Our data demonstrated that Piezo1 promote proliferation, invasion and migration of HCC cells through Smad2/3, the canonical TGF- $\beta$ signaling pathway.

Subsequently, we explored how did Piezo1 regulated TGF- $\beta$ signaling pathway. In present study, as the BioGrid database indicated, Piezo1 was identified to direct bonding to Rab5c, which is a small GTPase belongs to the Ras-superfamily. It has been shown that Piezo1 could recruits the small GTPase R-Ras to the endoplasmic reticulum (ER), had only a partial effect on H-Ras localization and no effect on other GTPase, in Chinese hamster ovary ( $\mathrm{CHO}$ ) cells, this process might relate to the C-terminus of R-RAS [36]. Normally, T $\beta R$ Il was internalized and a fraction of it was sorted from early endosomes to lysosomes for degradation [55]. Rab5c is a critical regulator of endosomes to lysosomes [56], which indicates that Pieoz1 might affect TGF- $\beta$ signaling through decreased degradation of T $\beta$ RII when Rab5c was recruited to membrane. Rab5c was determined to be involved in cell proliferation, transformation, survival and metastasis in types of cancers through various signaling pathways [33-35], and it was also upregulated in transcriptional level and was recruited more to cell membrane of Marfan vascular smooth muscle cells (VSMC) and activated TGF- $\beta$ signaling [24]. In this study, we found that Rab5c has a trend to located in membrane while Piezo1 highly expressed, then we confirmed that Rab5c was significantly enriched in membrane fractions of HCC cell lines. Therefore, our results indicated that Rab5c was the potential target of Piezo1 in HCC. In HCCLM3 ${ }^{\text {shPiezo1 }}$ and Hep3B ${ }^{\text {shPiezo1 }}$ cells, silencing of Piezo1 decreased activation of TGF- $\beta$ signaling. Meanwhile, Rab5c ectopic expression could regain the activity of TGF- $\beta$ signaling, and recovered the invasiveness, migrations, and mesenchymal phenotype in Piezo1 knockdown HCC cells. Although previous studies have confirmed that Rab5c was an oncogene that promote cancer progression or chemoresistance through various pathways [57-59], but our research first illustrate that Rab5c act as a target of Piezo1 and activate TGF- $\beta$ signaling through in cancer cells.

In conclusion, our study demonstrated that Piezo1 is highly expressed and is significantly correlated with poor prognosis. Piezo1 has potential to serve as a risk predicting marker for independent prognostic indicator for HCC patients. Moreover, we have identified that Piezo1 promoted HCC progression and EMT through activating TGF- $\beta$ signaling by recruiting Rab5c. Our finding also indicate that Piezo1 can be used as a novel prognostic biomarker and a potential therapeutic target in HCC.

\section{Declarations}

\section{Ethics approval and consent to participate:}

This study was ratified by the Ethics Committee of Committee of Xiangya Hospital, Central South University. All patients and their families provided written informed consent and agreed to the use of their tissue samples in the study in accordance with the Declaration of Helsinki. All the animal experiments approved by the Medical Experimental Animal Care Commission of CSU.

\section{Consent for publication:}

All authors have agreed to publish this manuscript. 
Availability of data and materials:

All data that support this study are available from the corresponding author upon reasonable request.

\section{Conflict of interest statement:}

No potential conflicts of interest are disclosed.

\section{Authorship contribution:}

Y.m.L. and L.y.Y. conceived the study and wrote the manuscript. Y.m.L., C.X. and B.S. conducted the experiments and contributed to the data analysis. Y.m.L., C.X., B.S. F.j.Z., M.m.C. and L.y.Y. collected clinical samples and corresponding clinical data. Y.m.L., and L.y.Y. revised the manuscript. All authors read and approved the final manuscript.

Funding: This work was supported by National Natural Science Foundation of China (81773139), Specialized Research Fund for Doctoral Program of Higher Education of China (20130162130007)

\section{Acknowledgements:}

Thank Professor Qiong-Qiong He and Professor Geng-Qiu Luo (Department of Pathology, Xiangya Hospital of Central South University) for the help of pathological diagnoses and guidance. Thank Professor Chao-Hui Zuo (The Affiliated Cancer Hospital of Xiangya School of Medicine) for helping to collect HCC specimens.

\section{References}

1. Bray F, Ferlay J, Soerjomataram I, et al. Global cancer statistics 2018: GLOBOCAN estimates of incidence and mortality worldwide for 36 cancers in 185 countries. CA Cancer J Clin 2018;68(6):394424. doi: 10.3322/caac.21492 [published Online First: 2018/09/13]

2. Zhou J, Sun H, Wang Z, et al. Guidelines for the Diagnosis and Treatment of Hepatocellular Carcinoma (2019 Edition). Liver Cancer 2020;9(6):682-720. doi: 10.1159/000509424 [published Online First: 2021/01/15]

3. Sun Y, Wu L, Zhong Y, et al. Single-cell landscape of the ecosystem in early-relapse hepatocellular carcinoma. Cel/ 2020 doi: 10.1016/j.cell.2020.11.041

4. Yang LY, Fang F, Ou DP, et al. Solitary large hepatocellular carcinoma: a specific subtype of hepatocellular carcinoma with good outcome after hepatic resection. Ann Surg 2009;249(1):118-23. doi: 10.1097/SLA.0b013e3181904988 [published Online First: 2008/12/25]

5. Tsilimigras DI, Mehta R, Paredes AZ, et al. Overall Tumor Burden Dictates Outcomes for Patients Undergoing Resection of Multinodular Hepatocellular Carcinoma Beyond the Milan Criteria. Annals of surgery 2020;272(4):574-81. doi: 10.1097/SLA.0000000000004346 
6. Luo YD, Fang L, Yu HQ, et al. p53 haploinsufficiency and increased mTOR signalling define a subset of aggressive hepatocellular carcinoma. J Hepatol 2021;74(1):96-108. doi:

10.1016/j.jhep.2020.07.036 [published Online First: 2020/08/02]

7. He K, Yan X, Li N, et al. Internalization of the TGF- $\beta$ type I receptor into caveolin-1 and EEA1 doublepositive early endosomes. Cell Research 2015;25(6):738-52. doi: 10.1038/cr.2015.60

8. Yang $H$, Fang $F$, Chang R, et al. MicroRNA-140-5p suppresses tumor growth and metastasis by targeting transforming growth factor beta receptor 1 and fibroblast growth factor 9 in hepatocellular carcinoma. Hepatology 2013;58(1):205-17. doi: 10.1002/hep.26315 [published Online First: 2013/02/13]

9. Xiao S, Chang RM, Yang MY, et al. Actin-like 6A predicts poor prognosis of hepatocellular carcinoma and promotes metastasis and epithelial-mesenchymal transition. Hepatology 2016;63(4):1256-71. doi: 10.1002/hep.28417 [published Online First: 2015/12/25]

10. Gao Q, Zhu H, Dong L, et al. Integrated Proteogenomic Characterization of HBV-Related Hepatocellular Carcinoma. Cell 2019;179(2) doi: 10.1016/j.cell.2019.08.052

11. Coste B, Mathur J, Schmidt M, et al. Piezo1 and Piezo2 are essential components of distinct mechanically activated cation channels. Science 2010;330(6000):55-60. doi:

10.1126/science. 1193270

12. Solis AG, Bielecki P, Steach HR, et al. Mechanosensation of cyclical force by PIEZO1 is essential for innate immunity. Nature 2019;573(7772):69-74. doi: 10.1038/s41586-019-1485-8

13. Liu Q, Sun B, Zhao J, et al. Increased Piezo1 channel activity in interstitial Cajal-like cells induces bladder hyperactivity by functionally interacting with NCX1 in rats with cyclophosphamide-induced cystitis. Experimental \& molecular medicine 2018;50(5) doi: 10.1038/s12276-018-0088-z

14. Romac JM, Shahid RA, Swain SM, et al. Piezo1 is a mechanically activated ion channel and mediates pressure induced pancreatitis. Nat Commun 2018;9(1):1715. doi: 10.1038/s41467-01804194-9 [published Online First: 2018/05/02]

15. Blythe NM, Muraki K, Ludlow MJ, et al. Mechanically activated Piezo1 channels of cardiac fibroblasts stimulate p38 mitogen-activated protein kinase activity and interleukin-6 secretion. J Biol Chem 2019;294(46):17395-408. doi: 10.1074/jbc.RA119.009167 [published Online First: 2019/10/06]

16. Li J, Hou B, Tumova S, et al. Piezo1 integration of vascular architecture with physiological force. Nature 2014;515(7526):279-82. doi: 10.1038/nature13701

17. Gudipaty SA, Lindblom J, Loftus PD, et al. Mechanical stretch triggers rapid epithelial cell division through Piezo1. Nature 2017;543(7643):118-21. doi: 10.1038/nature21407 [published Online First: 2017/02/16]

18. Pathak MM, Nourse JL, Tran T, et al. Stretch-activated ion channel Piezo1 directs lineage choice in human neural stem cells. Proc Natl Acad Sci U S A 2014;111(45):16148-53. doi:

10.1073/pnas. 1409802111

Page 13/31 
19. Choi D, Park E, Jung E, et al. Piezo1 incorporates mechanical force signals into the genetic program that governs lymphatic valve development and maintenance. JCl Insight 2019;4(5) doi:

10.1172/jci.insight.125068 [published Online First: 2019/01/25]

20. Zhang J, Zhou Y, Huang T, et al. PIEZO1 functions as a potential oncogene by promoting cell proliferation and migration in gastric carcinogenesis. Mol Carcinog 2018;57(9):1144-55. doi: 10.1002/mc.22831 [published Online First: 2018/04/24]

21. Li C, Rezania S, Kammerer S, et al. Piezo1 forms mechanosensitive ion channels in the human MCF7 breast cancer cell line. Sci Rep 2015;5:8364. doi: 10.1038/srep08364

22. Suzuki T, Muraki Y, Hatano N, et al. PIEZO1 Channel Is a Potential Regulator of Synovial Sarcoma Cell-Viability. Int J Mol Sci 2018;19(5) doi: 10.3390/ijms19051452 [published Online First: 2018/05/15]

23. Qu S, Li S, Hu Z. Upregulation of Piezo1 Is a Novel Prognostic Indicator in Glioma Patients. Cancer Manag Res 2020;12:3527-36. doi: 10.2147/CMAR.S251776 [published Online First: 2020/06/18]

24. Siegert AM, Serra-Peinado C, Gutierrez-Martinez E, et al. Altered TGF-beta endocytic trafficking contributes to the increased signaling in Marfan syndrome. Biochim Biophys Acta Mol Basis Dis 2018;1864(2):554-62. doi: 10.1016/j.bbadis.2017.11.015 [published Online First: 2017/11/28]

25. Wang JL, Yang MY, Xiao S, et al. Downregulation of castor zinc finger 1 predicts poor prognosis and facilitates hepatocellular carcinoma progression via MAPK/ERK signaling. J Exp Clin Cancer Res 2018;37(1):45. doi: 10.1186/s13046-018-0720-8 [published Online First: 2018/03/07]

26. Terpe HJ, Störkel S, Zimmer U, et al. Expression of CD44 isoforms in renal cell tumors. Positive correlation to tumor differentiation. Am J Patho/ 1996;148(2):453-63.

27. Liu L, Dai Y, Chen J, et al. Maelstrom promotes hepatocellular carcinoma metastasis by inducing epithelial-mesenchymal transition by way of Akt/GSK-3ß/Snail signaling. Hepatology 2014;59(2):531-43. doi: https://doi.org/10.1002/hep.26677

28. Fang $F$, Chang RM, Yu L, et al. MicroRNA-188-5p suppresses tumor cell proliferation and metastasis by directly targeting FGF5 in hepatocellular carcinoma. J Hepato/ 2015;63(4):874-85. doi: 10.1016/j.jhep.2015.05.008 [published Online First: 2015/05/23]

29. Chang RM, Yang H, Fang F, et al. MicroRNA-331-3p promotes proliferation and metastasis of hepatocellular carcinoma by targeting $\mathrm{PH}$ domain and leucine-rich repeat protein phosphatase. Hepatology 2014;60(4):1251-63. doi: 10.1002/hep.27221 [published Online First: 2014/05/16]

30. Chang RM, Xiao S, Lei X, et al. miRNA-487a Promotes Proliferation and Metastasis in Hepatocellular Carcinoma. Clin Cancer Res 2017;23(10):2593-604. doi: 10.1158/1078-0432.CCR-16-0851 [published Online First: 2016/11/09]

31. Liu H, Bian W, Yang D, et al. Inhibiting the Piezo1 channel protects microglia from acute hyperglycaemia damage through the JNK1 and mTOR signalling pathways. Life Sci 2021;264:118667. doi: 10.1016/j.Ifs.2020.118667 [published Online First: 2020/11/01]

32. Bagriantsev SN, Gracheva EO, Gallagher PG. Piezo proteins: regulators of mechanosensation and other cellular processes. J Biol Chem 2014;289(46):31673-81. doi: 10.1074/jbc.R114.612697 
[published Online First: 2014/10/12]

33. Saotome K, Murthy SE, Kefauver JM, et al. Structure of the mechanically activated ion channel Piezo1. Nature 2018;554(7693):481-86. doi: 10.1038/nature25453 [published Online First: 2017/12/21]

34. Yang XN, Lu YP, Liu JJ, et al. Piezo1 is as a novel trefoil factor family 1 binding protein that promotes gastric cancer cell mobility in vitro. Dig Dis Sci 2014;59(7):1428-35. doi: 10.1007/s10620-014-3044-3 [published Online First: 2014/05/07]

35. Aykut B, Chen R, Kim Jl, et al. Targeting Piezo1 unleashes innate immunity against cancer and infectious disease. Sci Immunol 2020;5(50) doi: 10.1126/sciimmunol.abb5168

36. McHugh BJ, Buttery R, Lad Y, et al. Integrin activation by Fam38A uses a novel mechanism of R-Ras targeting to the endoplasmic reticulum. J Cell Sci 2010;123(Pt 1):51-61. doi: 10.1242/jcs.056424 [published Online First: 2009/12/18]

37. Wang X, Wu WKK, Gao J, et al. Autophagy inhibition enhances PD-L1 expression in gastric cancer. $J$ Exp Clin Cancer Res 2019;38(1):140. doi: 10.1186/s13046-019-1148-5

38. Hasegawa K, Fujii S, Matsumoto S, et al. YAP signaling induces PIEZO1 to promote oral squamous cell carcinoma cell proliferation. The Journal of Pathology 2020 doi: 10.1002/path.5553

39. Sun Y, Li M, Liu G, et al. The function of Piezo1 in colon cancer metastasis and its potential regulatory mechanism. J Cancer Res Clin Oncol 2020;146(5):1139-52. doi: 10.1007/s00432-02003179-w [published Online First: 2020/03/11]

40. Cai Q, Wang S, Jin L, et al. Long non-coding RNA GBCDRInc1 induces chemoresistance of gallbladder cancer cells by activating autophagy. Molecular cancer 2019;18(1):82. doi: 10.1186/s12943-019-1016-0

41. Wang W, Yang LY, Huang GW, et al. Genomic analysis reveals RhoC as a potential marker in hepatocellular carcinoma with poor prognosis. Br J Cancer 2004;90(12):2349-55.

42. Yang L-Y, Wang W, Peng J-X, et al. Differentially expressed genes between solitary large hepatocellular carcinoma and nodular hepatocellular carcinoma. World J Gastroenterol 2004;10(24):3569-73.

43. Yang L, Xu J, Ou D, et al. Hepatectomy for huge hepatocellular carcinoma: single institute's experience. World J Surg 2013;37(9):2189-96. doi: 10.1007/s00268-013-2095-5

44. Yang L-Y, Chang R-M, Lau W-Y, et al. Mesohepatectomy for centrally located large hepatocellular carcinoma: Indications, techniques, and outcomes. Surgery 2014;156(5):1177-87. doi: 10.1016/j.surg.2014.05.012

45. Zhao YR, Wang JL, Xu C, et al. HEG1 indicates poor prognosis and promotes hepatocellular carcinoma invasion, metastasis, and EMT by activating Wnt/beta-catenin signaling. Clin Sci (Lond) 2019;133(14):1645-62. doi: 10.1042/CS20190225 [published Online First: 2019/07/07]

46. Kang $\mathrm{H}$, Hong $\mathrm{Z}$, Zhong $\mathrm{M}$, et al. Piezo1 mediates angiogenesis through activation of MT1-MMP signaling. Am J Physiol, Cell Physiol 2019;316(1) doi: 10.1152/ajpcell.00346.2018 
47. Zhao L, Li J, Liu Y, et al. Flotillin1 promotes EMT of human small cell lung cancer via TGF-beta signaling pathway. Cancer Biol Med 2018;15(4):400-14. doi: 10.20892/j.issn.2095-3941.2018.0053 [published Online First: 2019/02/16]

48. Fuxe J, Karlsson M. TGF- $\beta$-induced epithelial-mesenchymal transition: a link between cancer and inflammation. Seminars in cancer biology 2012;22:455-61. doi: 10.1016/j.semcancer.2012.05.004

49. Thakur AK, Nigri J, Lac S, et al. TAp73 loss favors Smad-independent TGF-beta signaling that drives EMT in pancreatic ductal adenocarcinoma. Cell Death Differ 2016;23(8):1358-70. doi:

10.1038/cdd.2016.18 [published Online First: 2016/03/05]

50. Liu S, Xu X, Fang Z, et al. Piezo1 impairs hepatocellular tumor growth via deregulation of the MAPKmediated YAP signaling pathway. Cell Calcium 2021;95:102367. doi: 10.1016/j.ceca.2021.102367 [published Online First: 2021/02/22]

51. Seoane J, Gomis RR. TGF-beta Family Signaling in Tumor Suppression and Cancer Progression. Cold Spring Harb Perspect Biol 2017;9(12) doi: 10.1101/cshperspect.a022277 [published Online First: 2017/03/02]

52. Fattet L, Yang J. RREB1 Integrates TGF-beta and RAS Signals to Drive EMT. Dev Cell 2020;52(3):25960. doi: 10.1016/j.devcel.2020.01.020 [published Online First: 2020/02/13]

53. Xu J, Lamouille S, Derynck R. TGF-beta-induced epithelial to mesenchymal transition. Cell Res 2009;19(2):156-72. doi: 10.1038/cr.2009.5 [published Online First: 2009/01/21]

54. Syed V. TGF-beta Signaling in Cancer. J Cell Biochem 2016;117(6):1279-87. doi: 10.1002/jcb.25496 [published Online First: 2016/01/17]

55. Mitchell $\mathrm{H}$, Choudhury A, Pagano RE, et al. Ligand-dependent and -independent transforming growth factor-beta receptor recycling regulated by clathrin-mediated endocytosis and Rab11. Mol Biol Cell 2004;15(9):4166-78. doi: 10.1091/mbc.e04-03-0245 [published Online First: 2004/07/02]

56. Heng J, Lv P, Zhang Y, et al. Rab5c-mediated endocytic trafficking regulates hematopoietic stem and progenitor cell development via Notch and AKT signaling. PLoS Biol 2020;18(4):e3000696. doi: 10.1371/journal.pbio.3000696 [published Online First: 2020/04/11]

57. Mendoza P, Ortiz R, Díaz J, et al. Rab5 activation promotes focal adhesion disassembly, migration and invasiveness in tumor cells. J Cell Sci 2013;126(Pt 17):3835-47. doi: 10.1242/jcs.119727

58. Onodera Y, Nam J-M, Hashimoto A, et al. Rab5c promotes AMAP1-PRKD2 complex formation to enhance $\beta 1$ integrin recycling in EGF-induced cancer invasion. J Cell Biol 2012;197(7):983-96. doi: $10.1083 /$ jcb.201201065

59. Takeda M, Koseki J, Takahashi H, et al. Disruption of Endolysosomal RAB5/7 Efficiently Eliminates Colorectal Cancer Stem Cells. Cancer Res 2019;79(7):1426-37. doi: 10.1158/0008-5472.CAN-18-2192

\section{Tables}

Table 1. Correlation between Piezo1 expression and clinicopathologic characteristics of HCC patients in training cohort and validation cohort. 


\begin{tabular}{|c|c|c|c|c|c|c|c|c|}
\hline \multirow{4}{*}{$\begin{array}{l}\text { Clinicopathologic } \\
\text { variables }\end{array}$} & \multicolumn{4}{|c|}{ Training cohort } & \multicolumn{4}{|c|}{ Validation cohort } \\
\hline & \multirow[t]{3}{*}{ n } & \multirow{2}{*}{\multicolumn{2}{|c|}{$\begin{array}{l}\text { Piezo1 } \\
\text { expression }\end{array}$}} & \multirow[t]{3}{*}{$P$} & \multirow[t]{3}{*}{$\mathrm{n}$} & \multirow{2}{*}{\multicolumn{2}{|c|}{ Piezo1 expression }} & \multirow[t]{3}{*}{$P$} \\
\hline & & & & & & & & \\
\hline & & High & Low & & & High & Low & \\
\hline Gender & & & & 0.190 & & & & 0.438 \\
\hline Female & 42 & 29 & 13 & & 31 & 17 & 14 & \\
\hline Male & 108 & 62 & 46 & & 99 & 62 & 37 & \\
\hline Age (years) & & & & 0.220 & & & & 0.238 \\
\hline$\leq 50$ & 67 & 37 & 30 & & 63 & 35 & 28 & \\
\hline$>50$ & 83 & 54 & 29 & & 67 & 44 & 23 & \\
\hline AFP(ng/ml) & & & & 0.266 & & & & 0.431 \\
\hline$<20$ & 36 & 19 & 17 & & 38 & 22 & 16 & \\
\hline$\geq 20$ & 114 & 72 & 42 & & 92 & 57 & 35 & \\
\hline Hepatitis B status & & & & 0.688 & & & & 0.496 \\
\hline Negative & 43 & 25 & 18 & & 48 & 31 & 17 & \\
\hline Positive & 107 & 66 & 41 & & 82 & 48 & 34 & \\
\hline Liver cirrhosis & & & & 0.196 & & & & 0.605 \\
\hline Absent & 69 & 38 & 31 & & 55 & 32 & 23 & \\
\hline Present & 81 & 53 & 28 & & 75 & 47 & 28 & \\
\hline Child-Pugh classification & & & & 0.587 & & & & 0.416 \\
\hline A & 98 & 61 & 37 & & 81 & 42 & 39 & \\
\hline B & 52 & 30 & 22 & & 49 & 29 & 20 & \\
\hline Tumor size(cm) & & & & 0.024 & & & & 0.020 \\
\hline$\leq 5$ & 57 & 28 & 29 & & 55 & 27 & 28 & \\
\hline$>5$ & 93 & 63 & 30 & & 75 & 52 & 23 & \\
\hline Tumor nodule number & & & & 0.004 & & & & 0.027 \\
\hline
\end{tabular}




\begin{tabular}{|c|c|c|c|c|c|c|c|c|}
\hline Solitary & 72 & 35 & 37 & & 71 & 37 & 34 & \\
\hline Multiple ( $\geq 2)$ & 78 & 56 & 22 & & 59 & 42 & 17 & \\
\hline Capsulation formation & & & & 0.014 & & & & 0.004 \\
\hline Presence & 58 & 28 & 30 & & 56 & 26 & 30 & \\
\hline Absence & 92 & 63 & 29 & & 74 & 53 & 21 & \\
\hline Edmondson-Steiner grade & & & & 0.004 & & & & 0.013 \\
\hline$\nabla \& \bigotimes$ & 62 & 29 & 33 & & 54 & 26 & 28 & \\
\hline$\nabla \& \nabla$ & 88 & 62 & 26 & & 76 & 53 & 23 & \\
\hline Microvascular invasion & & & & 0.034 & & & & 0.016 \\
\hline Absence & 91 & 49 & 42 & & 83 & 44 & 39 & \\
\hline Presence & 59 & 42 & 17 & & 47 & 35 & 12 & \\
\hline Macrovascular invasion & & & & 0.032 & & & & 0.027 \\
\hline Absence & 122 & 69 & 53 & & 108 & 61 & 47 & \\
\hline Presence & 28 & 22 & 6 & & 22 & 18 & 4 & \\
\hline BCLC stage & & & & 0.003 & & & & 0.044 \\
\hline $0 \& A$ & 43 & 18 & 25 & & 45 & 22 & 23 & \\
\hline$B \& C$ & 107 & 73 & 34 & & 85 & 57 & 28 & \\
\hline TNM stage & & & & 0.004 & & & & 0.029 \\
\hline \\
&\ & 62 & 29 & 33 & & 56 & 28 & 28 & \\
\hline \\
&\ & 88 & 62 & 26 & & 74 & 51 & 23 & \\
\hline CNLC stage & & & & 0.037 & & & & 0.003 \\
\hline$\nabla \& \nabla$ & 86 & 46 & 40 & & 81 & 41 & 40 & \\
\hline$\otimes \& \bigotimes$ & 64 & 45 & 19 & & 49 & 38 & 11 & \\
\hline
\end{tabular}

Abbreviations: AFP, alpha-fetoprotein; TNM, tumor node metastasis; BCLC, Barcelona Clinic Liver Cancer; CNLC, China Clinic Liver Cancer.

Table2. Univariate and multivariate analyses of risk factors associated with overall survival and diseasefree survival of HCC patients in training cohort. 


\begin{tabular}{|c|c|c|c|c|}
\hline \multirow{4}{*}{$\begin{array}{l}\text { Clinicopatholo-gic } \\
\text { variables }\end{array}$} & \multicolumn{2}{|l|}{ os } & \multicolumn{2}{|l|}{ DFS } \\
\hline & $\begin{array}{l}\text { Univariate } \\
\text { Analysis }\end{array}$ & $\begin{array}{l}\text { Multivariate } \\
\text { Analysis }\end{array}$ & $\begin{array}{l}\text { Univariate } \\
\text { Analysis }\end{array}$ & $\begin{array}{l}\text { Multivariate } \\
\text { Analysis }\end{array}$ \\
\hline & $P$ & $P$ & $P$ & $P$ \\
\hline & $\mathrm{HR}(95 \% \mathrm{Cl})$ & $\mathrm{HR}(95 \% \mathrm{Cl})$ & $\mathrm{HR}(95 \% \mathrm{Cl})$ & $\mathrm{HR}(95 \% \mathrm{Cl})$ \\
\hline Gender & 0.221 & NA & 0.245 & NA \\
\hline Female & 1 & & 1 & \\
\hline Male & $\begin{array}{l}1.295(0.856- \\
1.958)\end{array}$ & & $\begin{array}{l}1.278(0.845- \\
1.931)\end{array}$ & \\
\hline Age (years) & 0.018 & 0.325 & 0.014 & 0.113 \\
\hline$\leq 50$ & 1 & 1 & 1 & 1 \\
\hline$>50$ & $\begin{array}{l}1.595(1.085- \\
2.345)\end{array}$ & $\begin{array}{l}1.237(0.810- \\
1.889)\end{array}$ & $\begin{array}{l}1.622(1.102- \\
2.385)\end{array}$ & $\begin{array}{l}1.366(0.929- \\
2.008)\end{array}$ \\
\hline AFP(ng/ml) & 0.001 & 0.003 & 0.002 & $<0.001$ \\
\hline$<20$ & 1 & 1 & 1 & 1 \\
\hline$\geq 20$ & $\begin{array}{l}2.235(1.384- \\
3.611)\end{array}$ & $\begin{array}{l}2.198(1.300- \\
3.713)\end{array}$ & $\begin{array}{l}2.154(1.334- \\
3.478)\end{array}$ & $\begin{array}{l}2.308(1.441- \\
3.696)\end{array}$ \\
\hline Hepatitis B status & 0.655 & NA & 0.634 & NA \\
\hline Negative & 1 & & 1 & \\
\hline Positive & $\begin{array}{l}1.099(0.727- \\
1.660)\end{array}$ & & $\begin{array}{l}1.106(0.732- \\
1.671)\end{array}$ & \\
\hline Liver cirrhosis & 0.266 & NA & 0.350 & NA \\
\hline Absent & 1 & & 1 & \\
\hline Present & $\begin{array}{l}1.239(0.849- \\
1.809)\end{array}$ & & $\begin{array}{l}1.196(0.822- \\
1.739)\end{array}$ & \\
\hline $\begin{array}{l}\text { Child-Pugh } \\
\text { classification }\end{array}$ & 0.480 & NA & 0.468 & NA \\
\hline A & 1 & & 1 & \\
\hline$B$ & $\begin{array}{l}1.148(0.783- \\
1.684)\end{array}$ & & $\begin{array}{l}1.153(0.786- \\
1.691)\end{array}$ & \\
\hline Tumor size (cm) & 0.245 & NA & 0.267 & NA \\
\hline$\leq 5$ & 1 & & 1 & \\
\hline$>5$ & $\begin{array}{l}1.248(0.859- \\
1.814)\end{array}$ & & $\begin{array}{l}1.235(0.850- \\
1.795)\end{array}$ & \\
\hline
\end{tabular}




\begin{tabular}{|c|c|c|c|c|}
\hline Tumor nodule number & 0.001 & 0.001 & 0.001 & 0.002 \\
\hline Solitary & 1 & 1 & 1 & 1 \\
\hline Multiple ( $\geq 2$ ) & $\begin{array}{l}1.935(1.329- \\
2.817)\end{array}$ & $\begin{array}{l}2.195(1.379- \\
3.493)\end{array}$ & $\begin{array}{l}1.864(1.282- \\
2.710)\end{array}$ & $\begin{array}{l}1.983(1.296- \\
3.034)\end{array}$ \\
\hline Capsulation formation & 0.008 & 0.263 & 0.010 & 0.214 \\
\hline Presence & 1 & 1 & 1 & 1 \\
\hline Absence & $\begin{array}{l}1.650(1.139- \\
2.390)\end{array}$ & $\begin{array}{l}1.288(0.827- \\
2.005)\end{array}$ & $\begin{array}{l}1.619(1.120- \\
2.342)\end{array}$ & $\begin{array}{l}1.298(0.860- \\
1.960)\end{array}$ \\
\hline $\begin{array}{l}\text { Edmondson-Steiner } \\
\text { grade }\end{array}$ & 0.004 & 0.504 & 0.001 & 0.193 \\
\hline$\nabla \& \otimes$ & 1 & 1 & 1 & 1 \\
\hline$\varangle \& \bigotimes$ & $\begin{array}{l}1.784(1.207- \\
2.638)\end{array}$ & $\begin{array}{l}1.253(0.647- \\
2.427)\end{array}$ & $\begin{array}{l}1.790(1.253- \\
2.557)\end{array}$ & $\begin{array}{l}1.308(0.873- \\
1.958)\end{array}$ \\
\hline $\begin{array}{l}\text { Microvascular } \\
\text { invasion }\end{array}$ & 0.027 & 0.041 & $<0.001$ & 0.010 \\
\hline Absence & 1 & 1 & 1 & 1 \\
\hline Presence & $\begin{array}{l}1.529(1.049- \\
2.229)\end{array}$ & $\begin{array}{l}1.898(1.028- \\
3.507)\end{array}$ & $\begin{array}{l}2.110(1.489- \\
2.991)\end{array}$ & $\begin{array}{l}2.044(1.187- \\
3.522)\end{array}$ \\
\hline $\begin{array}{l}\text { Macrovascular } \\
\text { invasion }\end{array}$ & $<0.001$ & 0.008 & $<0.001$ & 0.012 \\
\hline Absence & 1 & 1 & 1 & 1 \\
\hline Presence & $\begin{array}{l}2.728(1.741- \\
4.273)\end{array}$ & $\begin{array}{l}2.191(1.230- \\
3.902)\end{array}$ & $\begin{array}{l}2.247(1.450- \\
3.483)\end{array}$ & $\begin{array}{l}2.086(1.174- \\
3.707)\end{array}$ \\
\hline BCLC stage & 0.001 & 0.012 & 0.001 & 0.001 \\
\hline $0 \& A$ & 1 & 1 & 1 & 1 \\
\hline$B \& C$ & $\begin{array}{l}1.914(1.301- \\
2.815)\end{array}$ & $\begin{array}{l}1.697(1.125- \\
2.560)\end{array}$ & $\begin{array}{l}1.828(1.289- \\
2.592)\end{array}$ & $\begin{array}{l}1.873(1.286- \\
2.729)\end{array}$ \\
\hline TNM stage & $<0.001$ & 0.010 & $<0.001$ & 0.005 \\
\hline$\nabla \& \otimes$ & 1 & 1 & 1 & 1 \\
\hline$\nabla \& \nabla$ & $\begin{array}{l}2.197(1.481- \\
3.259)\end{array}$ & $\begin{array}{l}1.778(1.148- \\
2.755)\end{array}$ & $\begin{array}{l}1.975(1.387- \\
2.813)\end{array}$ & $\begin{array}{l}1.797(1.196- \\
2.700)\end{array}$ \\
\hline CNLC stage & $<0.001$ & 0.001 & 0.008 & 0.045 \\
\hline$\varangle \& \bigotimes$ & 1 & 1 & 1 & 1 \\
\hline$\nabla \& \nabla$ & $\begin{array}{l}2.300(1.576- \\
3.358)\end{array}$ & $\begin{array}{l}2.019(1.318- \\
3.094)\end{array}$ & $\begin{array}{l}1.593(1.130- \\
2.246)\end{array}$ & $\begin{array}{l}2.002(1.017- \\
3.942)\end{array}$ \\
\hline
\end{tabular}




\begin{tabular}{|lllll|} 
Piezo1 expression & $<0.001$ & 0.026 & $<0.001$ & 0.001 \\
\hline Low & 1 & 1 & 1 & 1 \\
\hline High & $2.048(1.374-$ & $1.671(1.064-$ & $1.943(1.355-$ & $1.894(1.285-$ \\
& $3.053)$ & $2.625)$ & $2.787)$ & $2.972)$ \\
\hline
\end{tabular}

Abbreviations: $\mathrm{HR}$, hazard risk ratio; $\mathrm{Cl}$, confidence interval; NA, not applicable, AFP, alpha-fetoprotein; HBsAg, hepatitis B surface antigen; TNM, tumor node metastasis; BCLC, Barcelona Clinic Liver Cancer; CNLC, China Clinic Liver Cancer.

Figures 


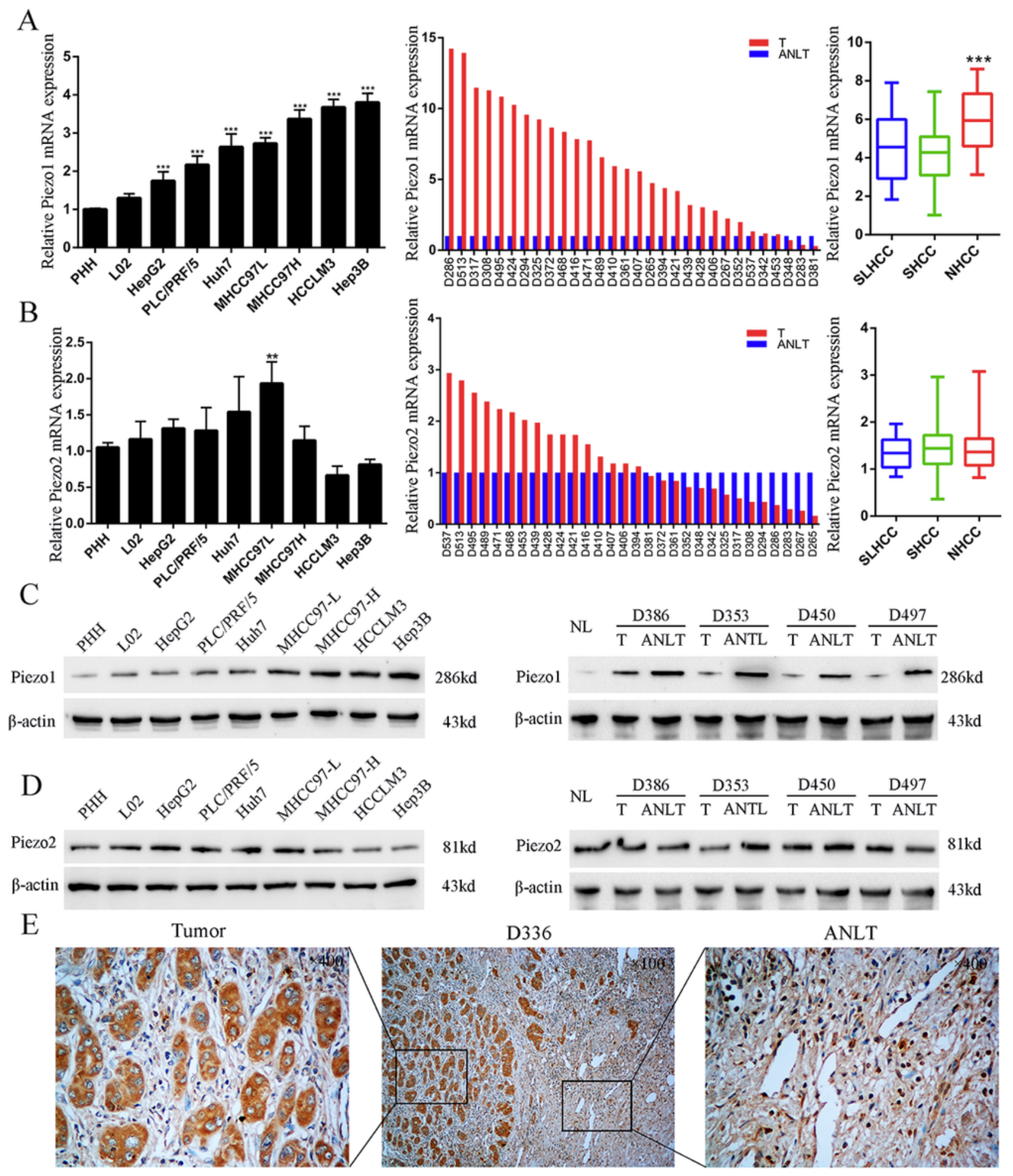

\section{Figure 1}

Piezo1 is significantly upregulated in HCC cell lines and tissues. (A-B) qRT-PCR identified the mRNA of Piezo1(A) and Piezo2 (B) in seven HCC cell lines, PHH and L02 cells, 30 pairs of fresh HCC tissues and ANLTs, and 3 clinical subtypes of HCC tissues. Data are shown as mean \pm SD. (C-D) Piezo1(C) and Piezo2(D) protein expression levels were detected in seven HCC cell lines, PHH and L02 cells, in normal liver tissue and paired fresh HCC tissues and ANLTs by Western blot. (E) Representative IHC images of 
Piezo1 expression in HCC tumor tissue and ANLTs. Abbreviations: PHH, Primary human hepatocytes; SLHCC, Solitary Large HCC; SHCC, Small HCC; NHCC, Nodular HCC; NL, Normal liver; T, HCC tissue; ANLT, Adjusted non-tumor liver tissue. *, $\mathrm{P}<0.05 ; * \star, \mathrm{P}<0.01 ; * \star \star, \mathrm{P}<0.001$.
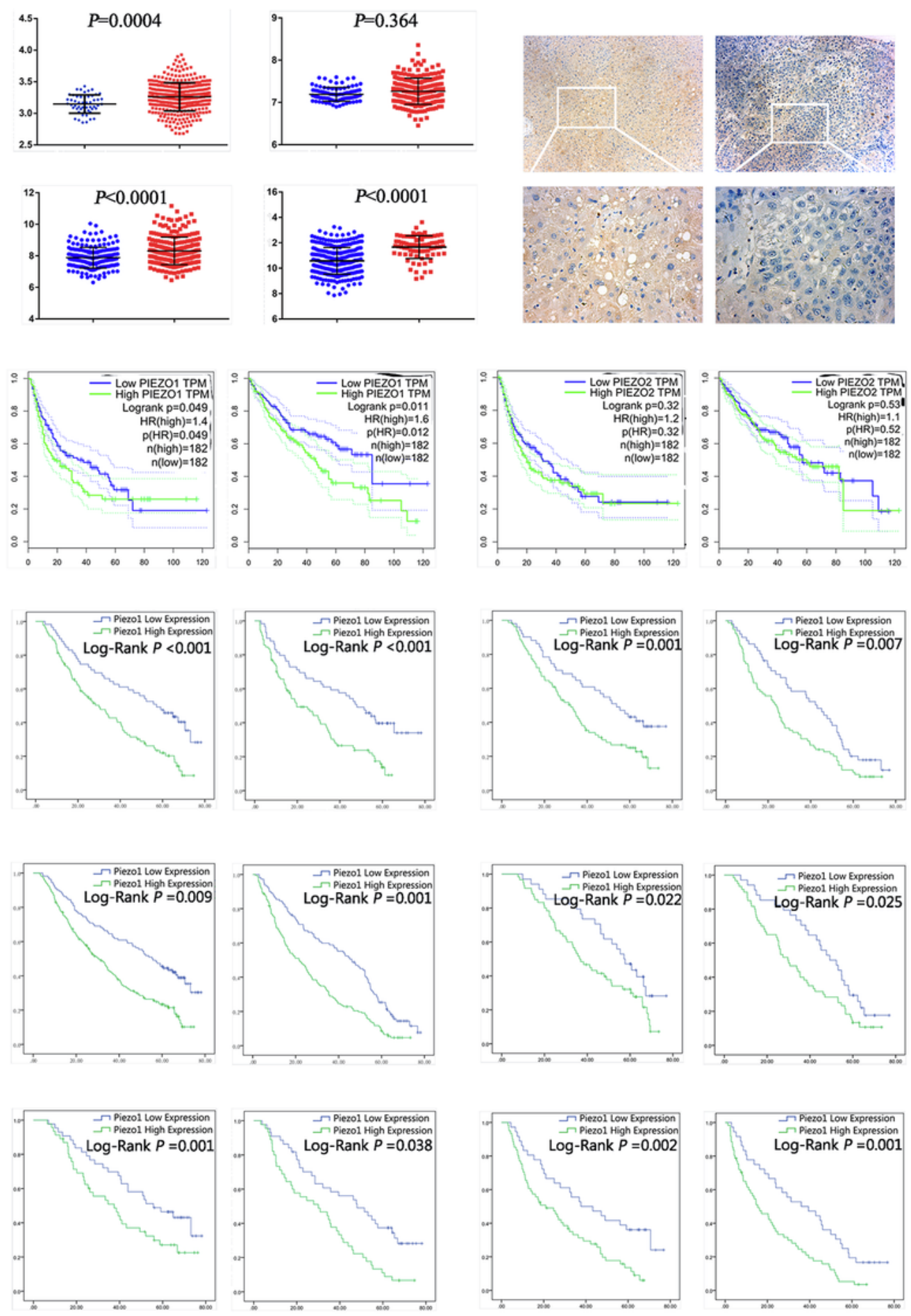

Figure 2

Upregulated Piezo1 is associated with HCC poor prognosis. (A) Piezo1 expression was higher in HCC tissues than Normal liver tissues according to the analysis of data from TCGA and GEO (GSE76297, 
GSE36376, GSE10143). (B) Representative images of low Piezo1 expression cases and high Piezo1 expression cases were shown. Magnification, $\times 100, \times 400$. (C) Kaplan-Meier analysis of OS and DFS based on Piezo1 and Piezo2 mRNA expression in data from TCGA. (D) Kaplan-Meier analysis of OS and DFS with high or low Piezo1 expression in Training and Validation Cohort. (E) The OS and DFS of HCC patients in the cohort integrated by Training and Validation cohort. The survival curve was calculated with the log-rank test in SLHCC, SHCC, and NHCC, the three clinical HCC subtypes.

A.

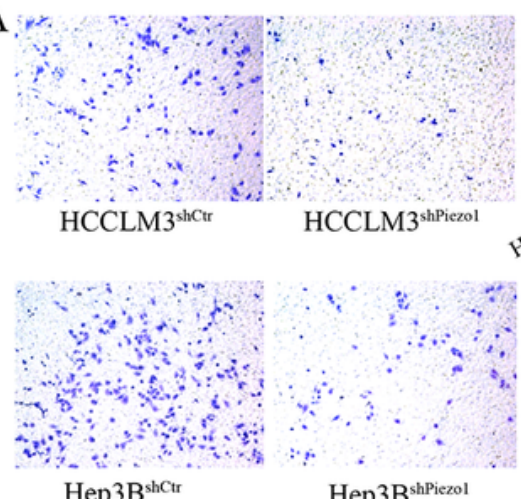

$\mathrm{C}$
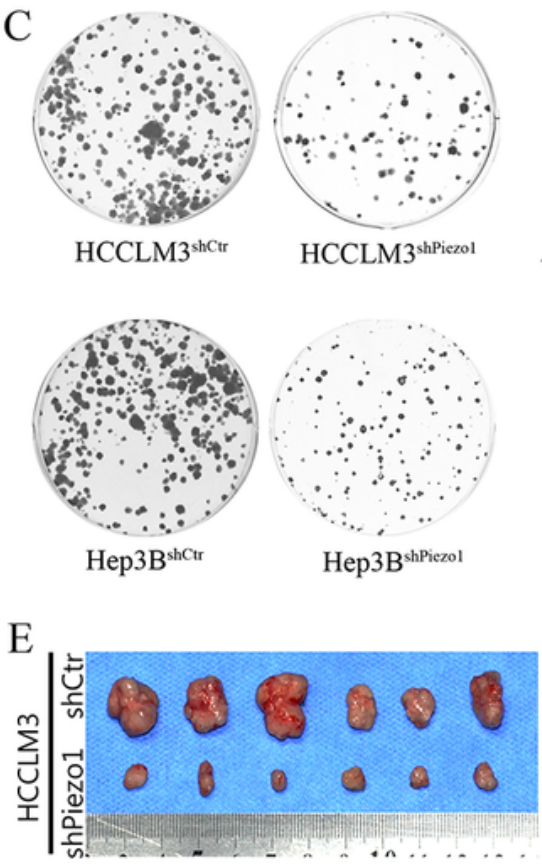

$\mathrm{F}$

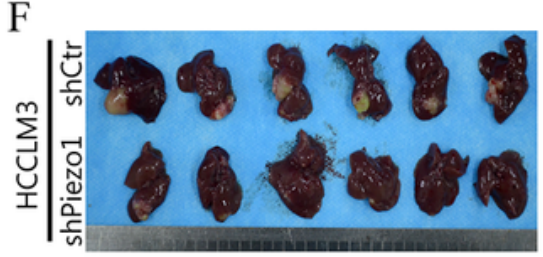

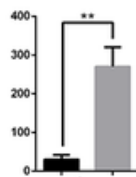
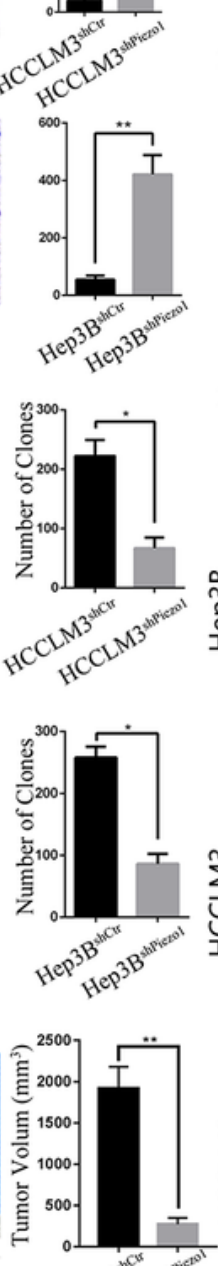

${ }_{1} \mathrm{CC}_{\mathrm{A}} \mathrm{CC}^{\mathrm{N}^{3}}$

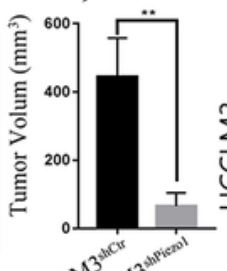

${ }_{1} \mathrm{CC}_{\mathrm{A}} \mathrm{CO}^{\mathrm{N}^{3}}$
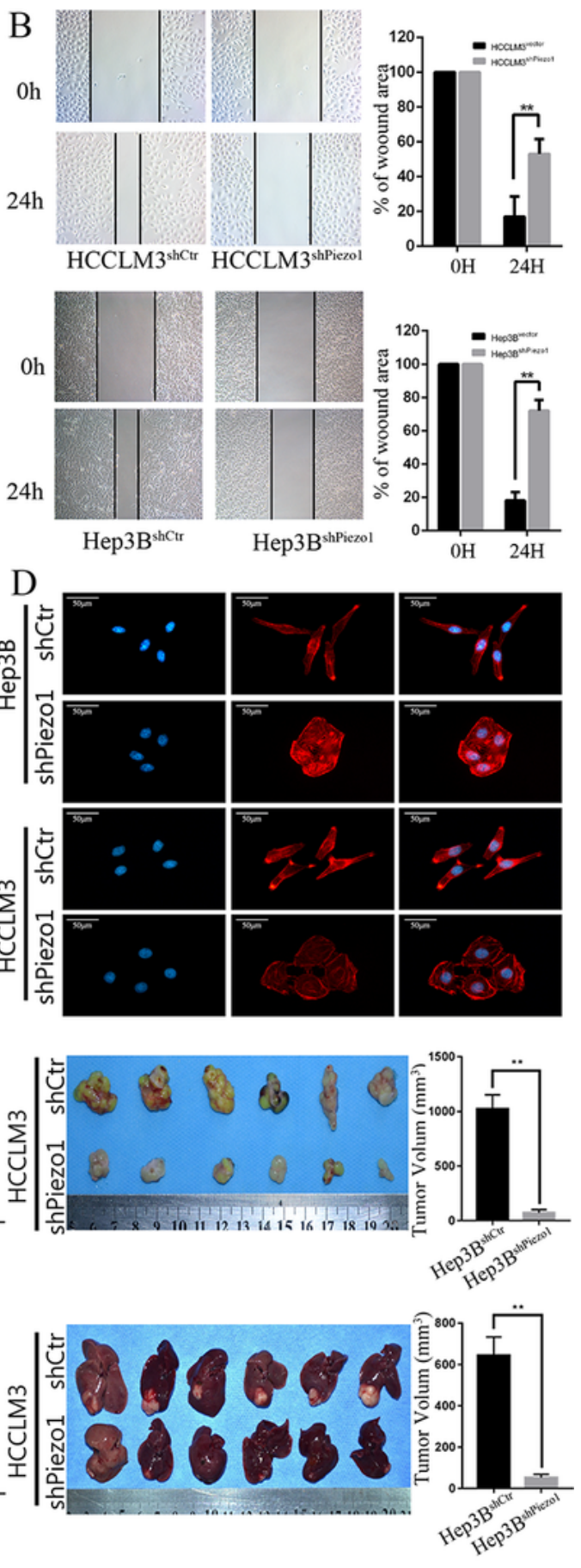
Piezo1 promotes HCC invasion, proliferation and metastasis in vitro and in vivo. (A-B) Transwell invasion assay (A) and Wound healing assay (B) were used to detect the invasive and migratory capacities of HCCLM3shPiezo1, Hep3BshPiezo1 and the corresponding control cells ( $n=6$ for each group). (C) Colony formation assays was used to detect the proliferation of Hep3BshPiezo1, HCCLM3shPiezo1 and the control HCC cells ( $n=6$ for each group). (D) Representative images of the cytoskeleton showed that Piezo1 affected the cellular morphology. (E) Subcutaneous tumors from HCCLM3shPiezo1 and Hep3BshPiezo1 cells and their control cells. Tumor volumes of tumors are shown in the right panels $(n=6$ for each group). (F) Orthotopic tumors from from HCCLM3shPiezo1 and Hep3BshPiezo1 cells and their control cells. Tumor weight are shown in the right panels ( $n=6$ for each group) ${ }^{\star}, P<0.05 ; \star \star, P<0.01$. 
A

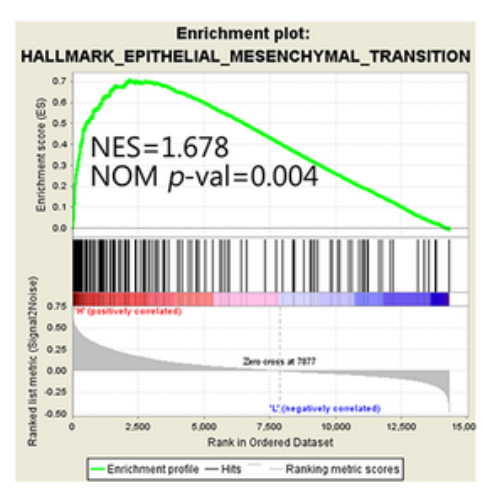

C

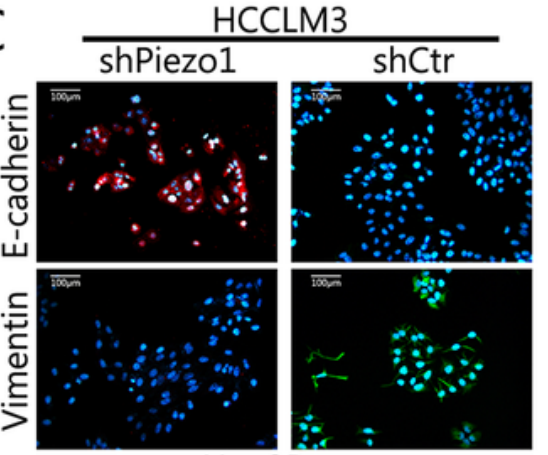

Hep3B
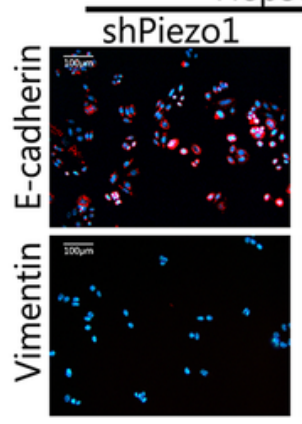

E
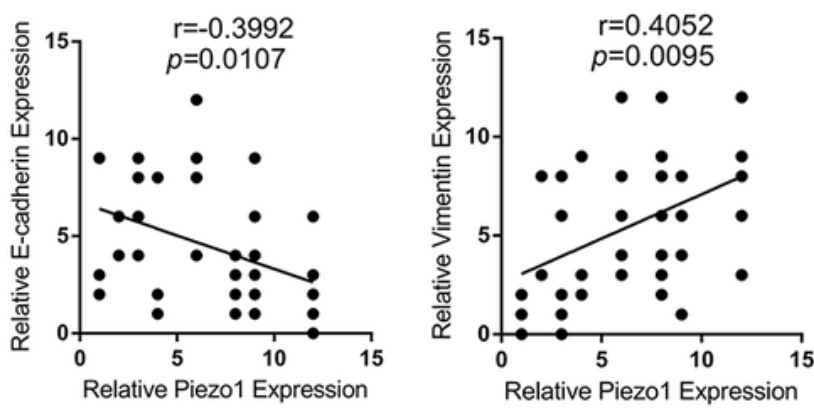

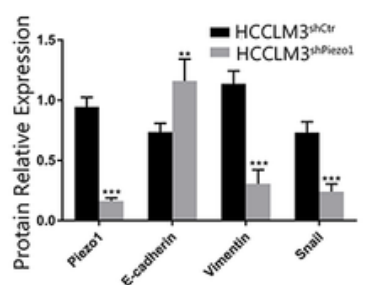

Vimentin _..

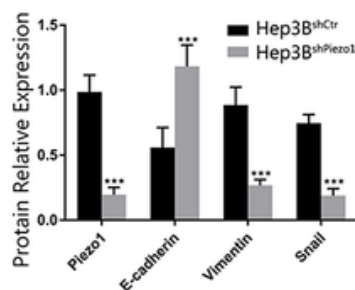

D461

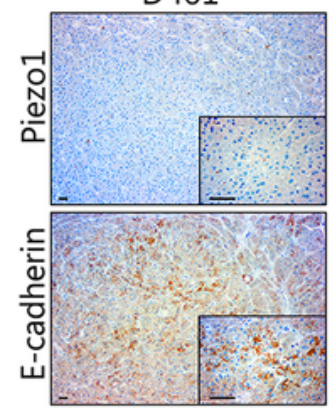

D353
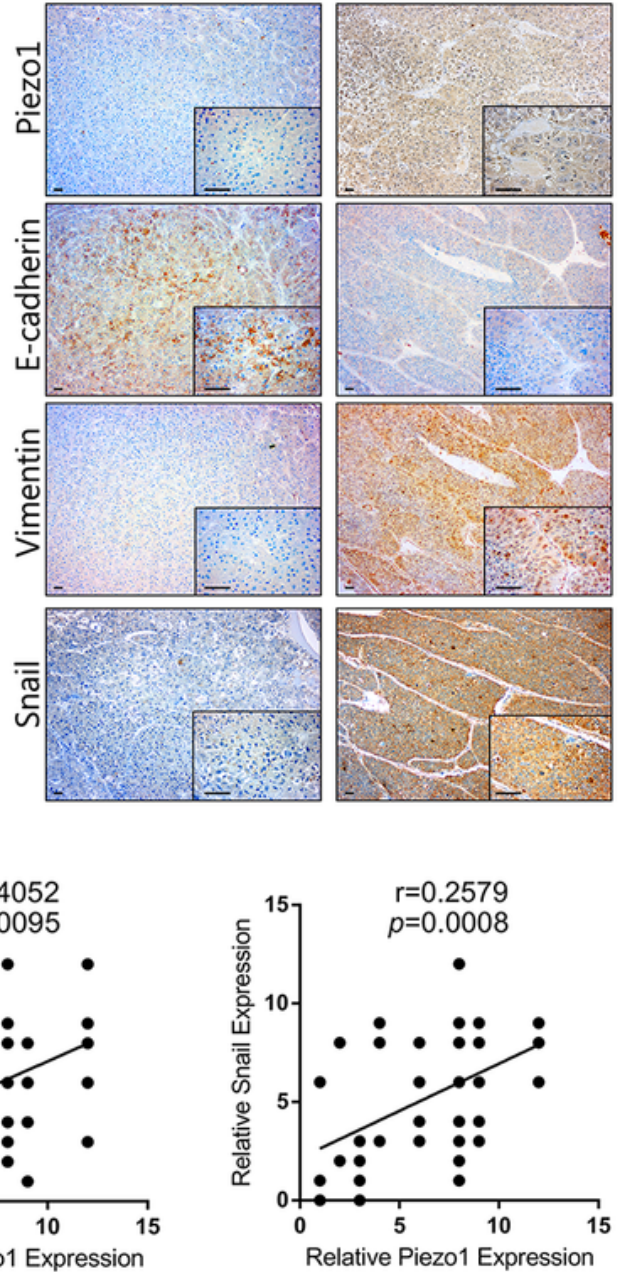

\section{Figure 4}

Piezo1 promotes EMT in HCC. (A) GSEA of Piezo1 expression in HCC patients from the TCGA database revealed that the EMT signature is significantly positively associated with Piezo1 expression (NES = 1.678, NOM p-val=0.004). (B) Piezo1-mediated expression levels of EMT markers were detected by western blot. The right panel showed the result of semi-quantitative analysis of western bolt. (C) Double immunofluorescence staining showed that Piezo1 affected cellular expression of EMT markers (Red: E- 
cadherin; Green: Vimentin). (D)Representative IHC images of Piezo1, E-cadherin, vimentin and Snail expression in HCC tissue. (E)Piezo1 and EMT markers expression correlations were analyzed by Spearman rank correlation tests in the Clinical samples( $n=40)$. **, $P<0.01 ; * \star *, P<0.001$.

A
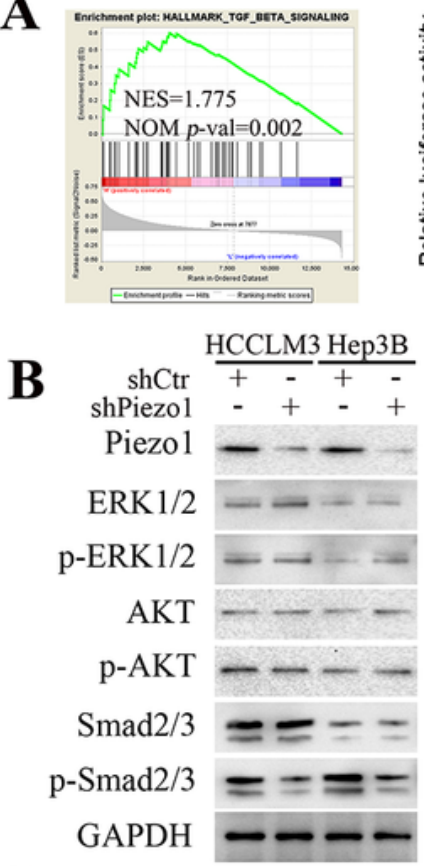

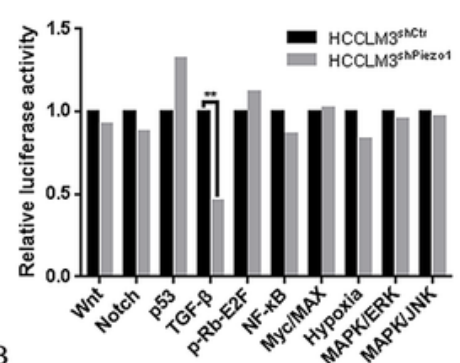

C
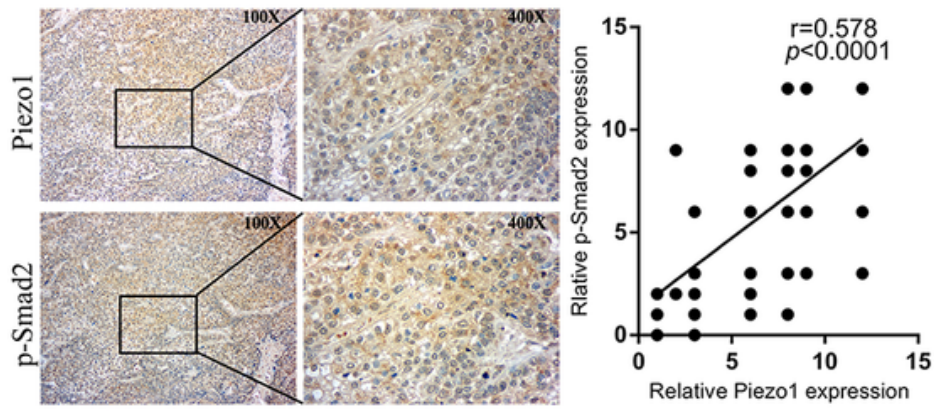

D
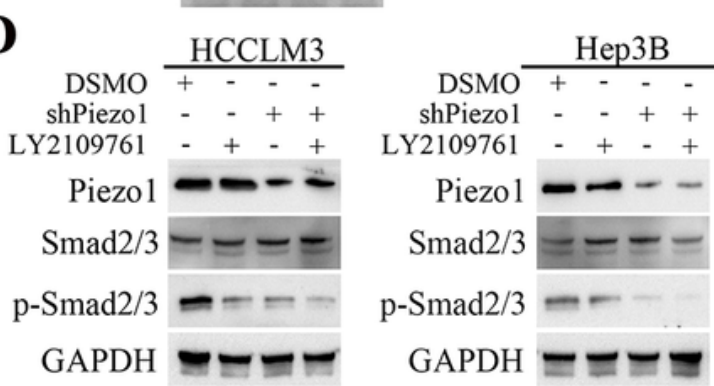

$\mathbf{F}$

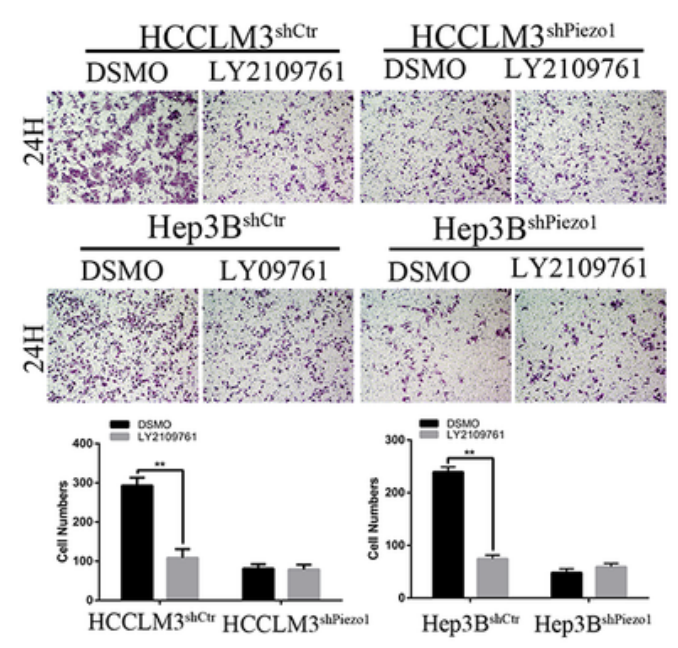

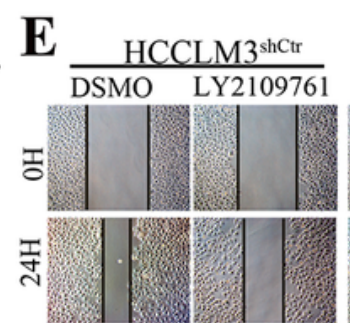
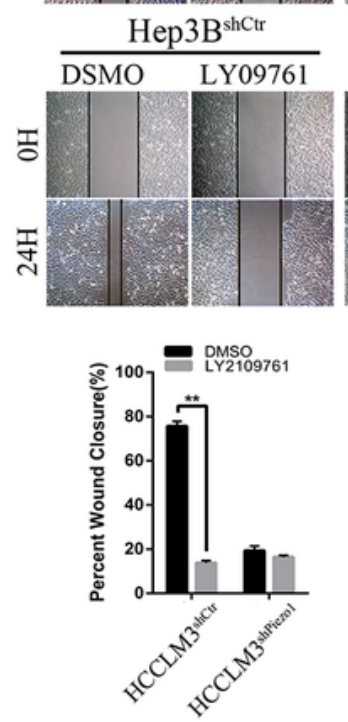
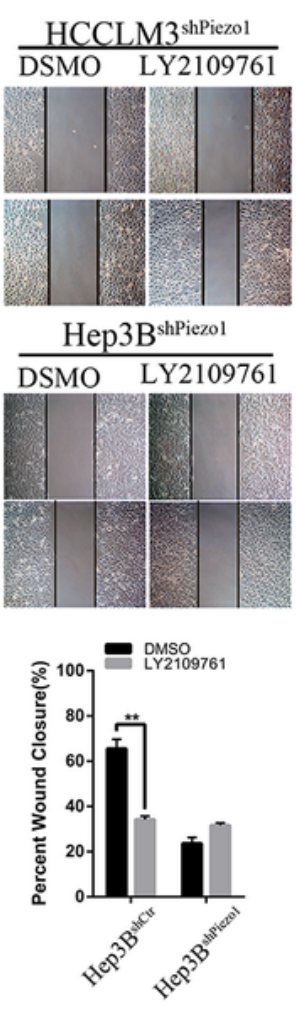

Figure 5

Piezo1 promote HCC growth and metastasis via TGF- $\beta$ signaling. (A)GSEA of Piezo1 expression in HCC patients from the TCGA database revealed that the TGF- $\beta$ signaling is significantly positively associated 
with Piezo1 expression (NES $=1.775$, NOM p-val=0.002). The right panels of 10-Pathway Reporter Array showed the TGF- $\beta$ signaling significantly changed in Piezo1 interfered cells. (B) The protein expression levels of Piezo1 and markers of TGF- $\beta$ signaling were detected by Western blot. (C) Representative IHC images of Piezo1 and p-Smad2, their expression correlations were analyzed by Spearman rank correlation tests in $40 \mathrm{HCC}$ tissue. (D) Smad2/3 and p-Smad2/3 in HCC cells after interfered Piezo1 or blocked TGF- $\beta$ signaling (Blocked by LY2109761, the inhibitor of TGF- $\beta$ pathway) were detected by WB. (E-F) Wound healing(E) and Transwell invasion(F) assays for HCCLM3shPiezo1, Hep3BshPiezo1 and their control cells with/without LY2109761 treatment. 
A

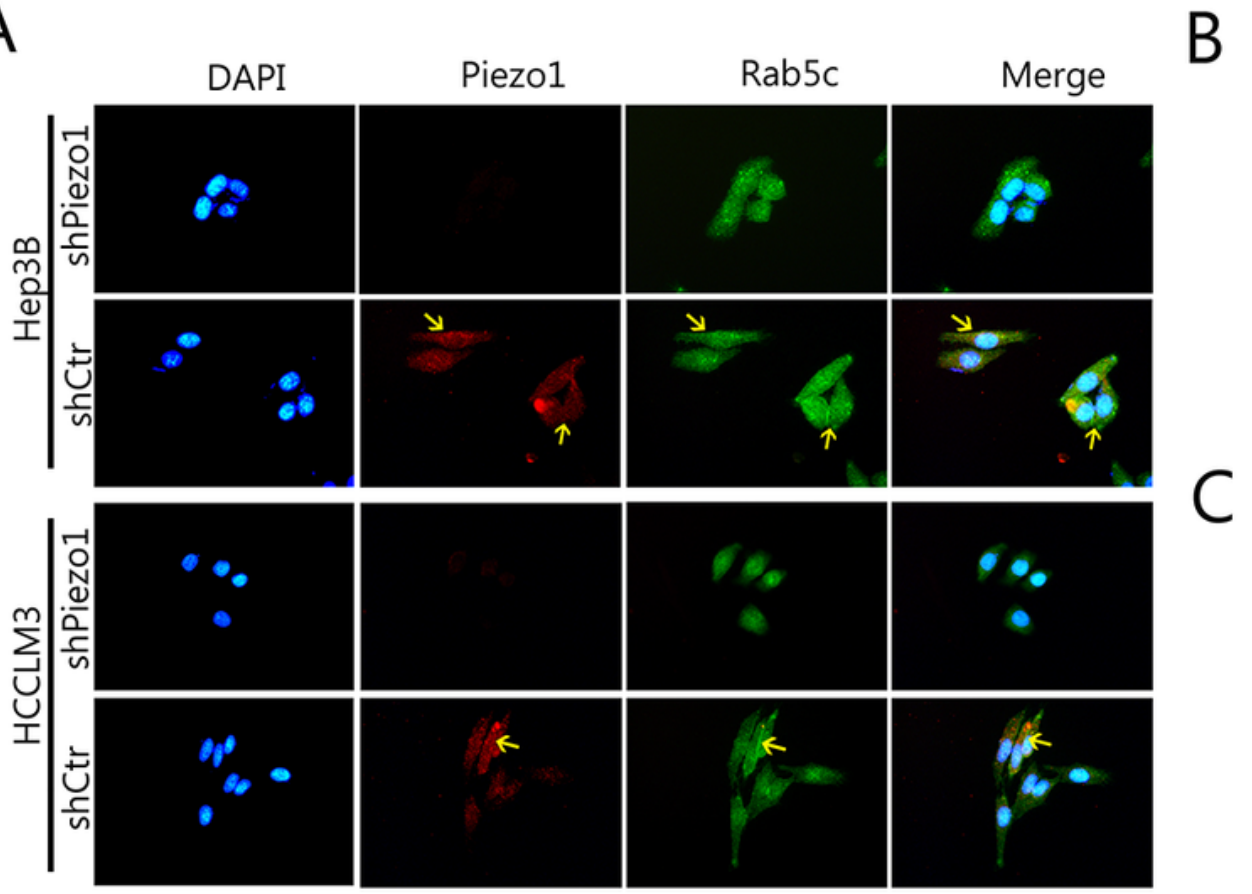

D

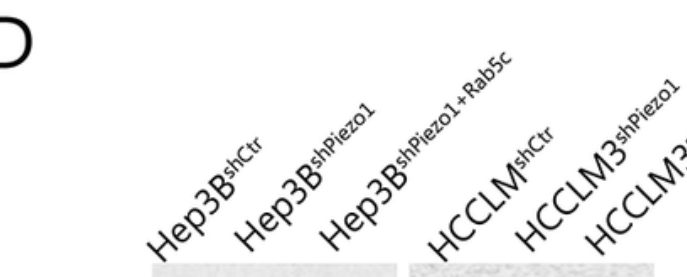

Piezo1
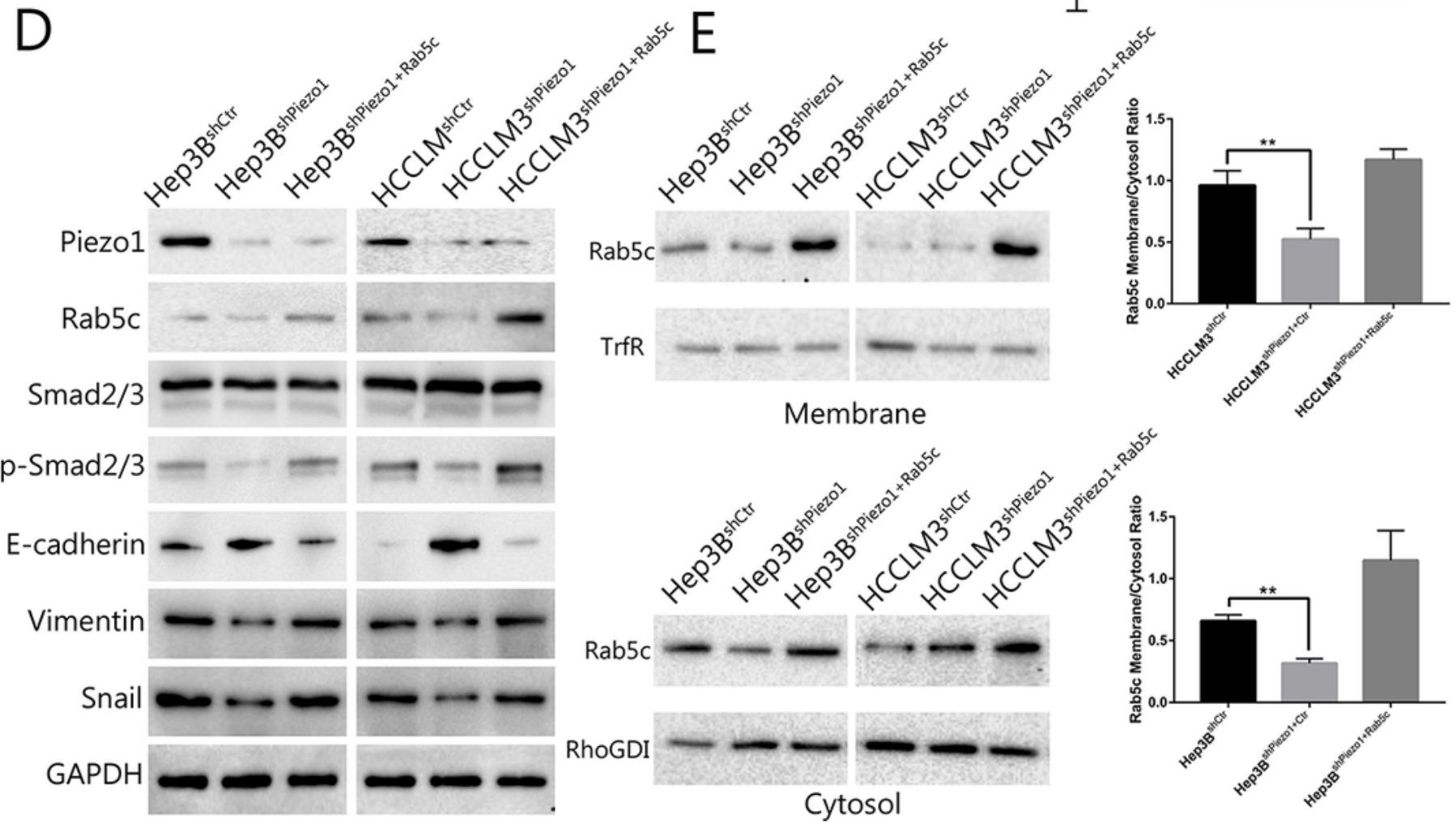

Membrane
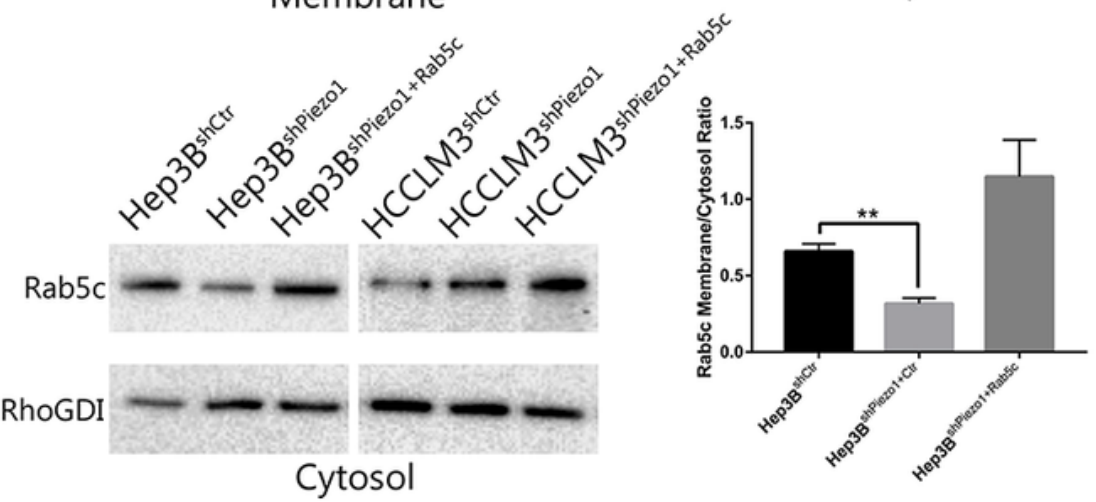

\section{Figure 6}

Piezo1 activates TGF- $\beta /$ Smad2/3 signaling by recruiting Rab5c. (A)Rab5c tend to located at membrane with the presence of Piezo1 revealed by Immunofluorescence (IF) co-localization. (B-C) Piezo1(B) and Rab5c(C) could interact with each other in HCC cells analyzed by Co-immunoprecipitation assay. (D) Protein expression level analyzed by western blot and showed that Piezo1 regulated the phosphorylation level of Smad2/3 and expression level of EMT markers, but not rab5c expression level. (E) Membrane 
enrichment analysis by Western blotting of RAB5c $(N=5, * \star p<<0.01)$. Membrane and cytosol fractions from HCC cells were obtained and analyzed by Western blot. Membrane and cytosol bands were relativized against their respective fraction marker, and membrane fractions were normalized against their corresponding cytosolic fraction.

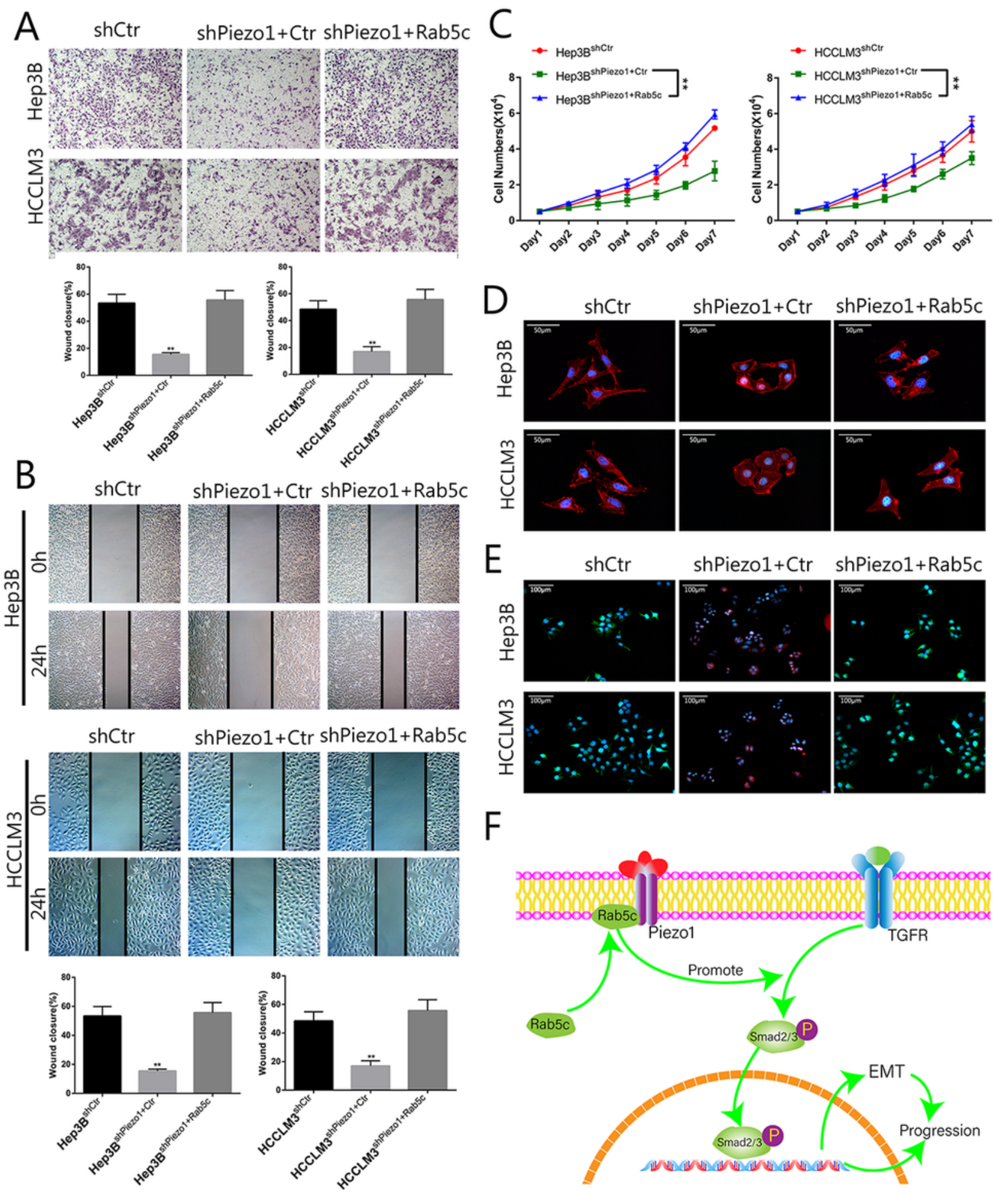

Figure 7 
Piezo1 mediated activating of TGF- $\beta$ signaling and promotion of HCC proliferation, metastasis and EMT through Rab5c. (A-B) Transwell invasion (A) and Wound healing assays (B) of HCC cells with interfered expression of piezo1 and ectopic expression of Rab5c. (C)Cytoskeleton and EMT marker expression assays of HCC cells with interfered expression of piezo1 and ectopic expression of Rab5c. (D) MTT assays were performed to test the influence of Rab5c on the proliferation of HCC cells with interfered expression of piezo1 and ectopic expression of Rab5c. (E) Schematic depiction of Piezo1 promotes progression of HCC activating through TGF- $\beta$ signaling by recruiting Rab5c. ${ }^{\star}, \mathrm{P}<0.01$.

\section{Supplementary Files}

This is a list of supplementary files associated with this preprint. Click to download.

- SupplementaryTables.docx

- SupplementaryFigures.pdf 